\title{
Diffusion Magnetic Resonance Imaging (dMRI)
}

\section{Jian Cheng}

Department of Radiology and Biomedical Imaging Research Institute, University of North Carolina at Chapel Hill

\section{Hongtu Zhu}

Department of Biostatistics and Biomedical Imaging Research Institute, University of North Carolina at Chapel Hill

\section{CONTENTS}

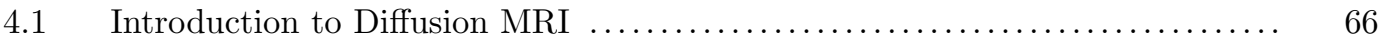

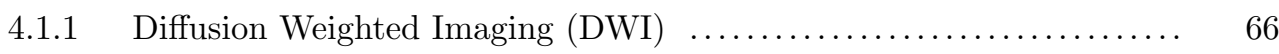

4.1.1.1 Diffusion Gradient Sequence $\ldots \ldots \ldots \ldots \ldots \ldots \ldots \ldots \ldots . \ldots 6$

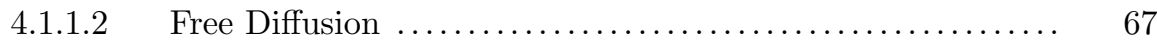

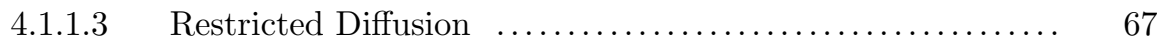

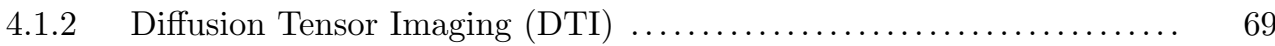

4.1.2.1 Scalar Indices and Eigenvectors of Diffusion Tensor ...... 69

4.2 High Angular Resolution Diffusion Imaging (HARDI) $\ldots \ldots \ldots \ldots \ldots \ldots \ldots \ldots . \ldots$

4.2.1 Generalization of Diffusion Tensor Imaging $\ldots \ldots \ldots \ldots \ldots \ldots \ldots \ldots \ldots \ldots$

4.2.1.1 Mixture of Tensor Model .......................... 70

4.2.1.2 Generalized DTI (GDTI) ....................... 71

4.2.1.3 High-Order Tensor Model, ADC-Based Model .......... 72

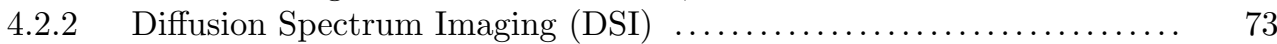

4.2 .3 Hybrid Diffusion Imaging (HYDI) $\ldots \ldots \ldots \ldots \ldots \ldots \ldots \ldots \ldots \ldots \ldots . \ldots \ldots$

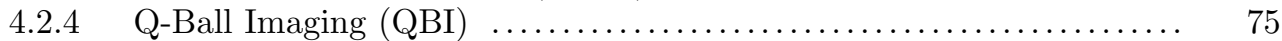

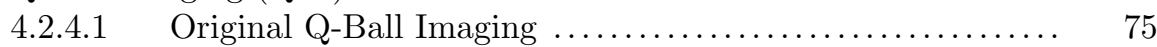

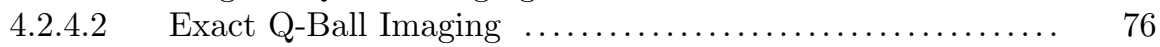

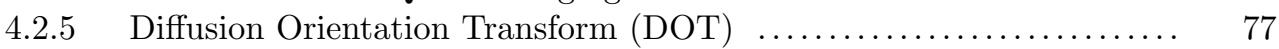

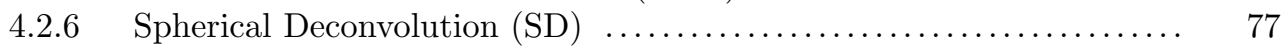

4.2.7 Diffusion Propagator Imaging (DPI) $\ldots \ldots \ldots \ldots \ldots \ldots \ldots \ldots \ldots \ldots \ldots \ldots \ldots \ldots$

4.2.8 Simple Harmonic Oscillator Reconstruction and Estimation (SHORE) 78

4.2.9 Spherical Polar Fourier Imaging (SPFI) $\ldots \ldots \ldots \ldots \ldots \ldots \ldots \ldots \ldots . \ldots \ldots$

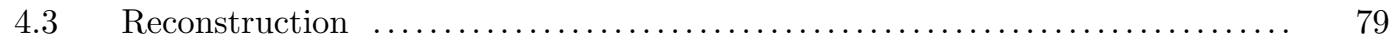

4.3.1 Noise Components and Voxelwise Estimation Methods ........... 79

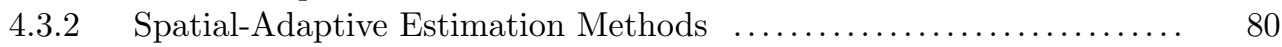

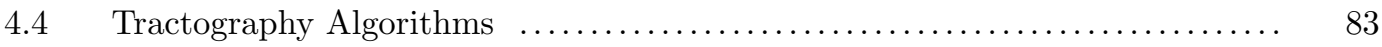

4.5 Uncertainty in Estimated Diffusion Quantities $\ldots \ldots \ldots \ldots \ldots \ldots \ldots \ldots \ldots \ldots$

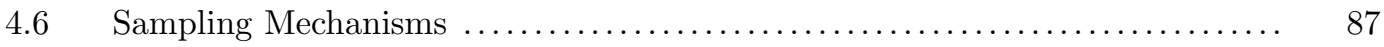

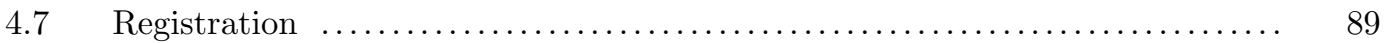

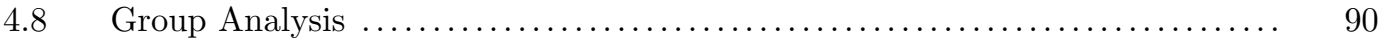

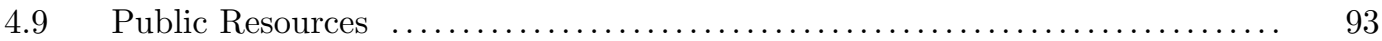

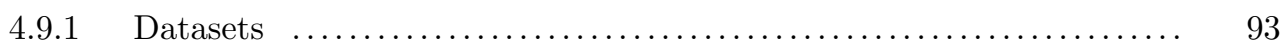

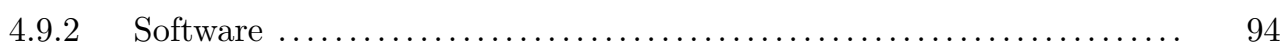




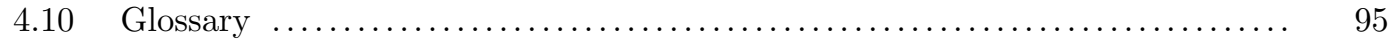

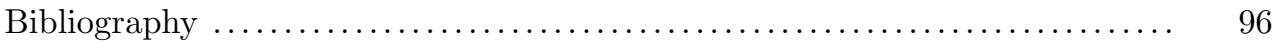

\subsection{Introduction to Diffusion MRI}

Since the 1980s, diffusion magnetic resonance imaging (dMRI) as a magnetic resonance imaging (MRI) technique has been widely used to track the effective diffusion of water molecules, which is hindered by many obstacles (e.g., fibers or membranes), in the human brain in vivo. Because water molecules tend to diffuse slowly across white matter fibers and diffuse fast along such fibers, the use of dMRI to track water diffusion allows one to map the microstructure and organization of those white matter pathways (17). Quantitatively measuring the diffusion process is critical for a quantitative assessment of the integrity of anatomical connectivity in white matter and its association with brain functional connectivity. Its clinical applications include normal brain maturation and aging, cerebral ischemia, multiple sclerosis, epilepsy, metabolic disorders, and brain tumors, among many others. Although there are several nice review papers and monographies on dMRI $(15,5,101,81)$, this chapter was written for the readers who are interested in the theoretical underpinning of various mathematical and statistical methods associated with dMRI. Due to limitations of space, we are unable to cite all important papers in the dMRI literature.

\subsubsection{Diffusion Weighted Imaging (DWI)}

\subsubsection{Diffusion Gradient Sequence}

In diffusion weighted imaging (DWI), imaging signals can be made sensitive to diffusion through some diffusion gradient sequences. A standard diffusion gradient sequence used in dMRI is the Pulsed Gradient Spin-Echo (PGSE) sequence proposed by Stejskal and Tanner (126). See Figure 4.1 for the sketch map of the PGSE sequence. The PGSE sequence consists of two gradient pulses $\mathbf{G}(t)$ with duration time $\delta$. Although it is common to use rectangular gradient lobes in the PGSE sequence, there are other kinds of gradient lobes commonly used in dMRI (24, chap. 9).

(i) The first 90 radio-frequency $(\mathrm{RF})$ pulse translates the spins into the transverse plane, i.e. the $x-y$ plane, considering the $\mathbf{B}_{0}$ is along the $z$-axis. Then the spins precess around $\mathbf{B}_{0}$ with RF $\omega_{0}$. Due to local magnetic field inhomogeneities, some spins slow down and some spins speed up. ${ }^{1}$

(ii) After time $\Delta$ between two pulses, the second $180 \mathrm{RF}$ pulse refocuses the phase of spins so that slower spins lead ahead and the fast ones trail behind. The spin echo process occurs when the spins recover their net magnetization.

(iii) The scanner coils receive the diffusion signal at echo time $t=T E$ after the two pulses.

Mathematically, the diffusion gradient $\mathbf{G}($ ) sequence can be written as

$$
\mathbf{G}(t)=H\left(t_{1}\right) \quad H\left(t_{1} \quad \delta\right)+H\left(t_{2}\right) \quad H\left(t_{2} \quad \delta\right) \mathbf{G},
$$

where $t_{2}=t_{1}+\Delta, H()$ is the heaviside step function, and $\mathbf{G}=\mathbf{G} \mathbf{u}$, in which $\mathbf{u} \quad S^{2}$ represents the gradient direction.

\footnotetext{
${ }^{1}$ http://en.wikipedia.org/wiki/Spin_echo
} 


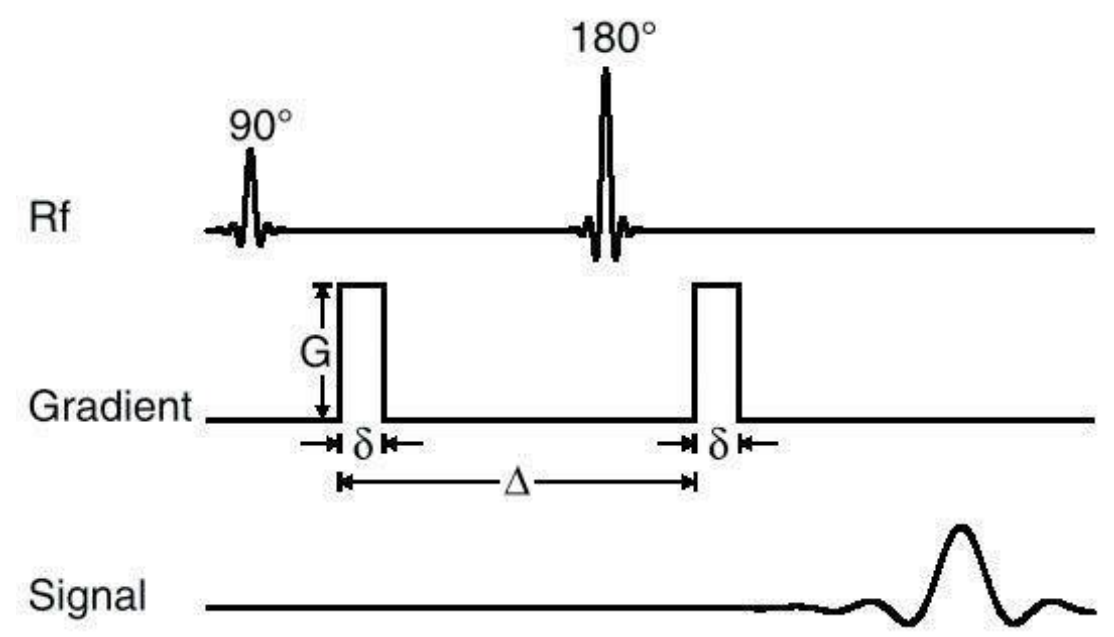

\section{FIGURE 4.1}

Pulsed Gradient Spin-Echo (PGSE) sequence introduced by Stejskal and Tanner (126). $\delta$ is the duration of the diffusion gradient pulses and $\Delta$ is the time between two diffusion gradient pulses.

\subsubsection{Free Diffusion}

Let $S(b)$ and $S(0)$ be, respectively, DWI signal at the diffusion weighting factor $b$ and at $b=0$, where $b$ is defined below. Given the PGSE sequence with the gradient sequence in (4.1), the diffusion weighted signal attenuation $E(b)=S(b) / S(0)$ is given by the Stejskal-Tanner equation (126)

$$
E(b)=\exp (b D),
$$

where $D$ is known as the Apparent Diffusion Coefficient (ADC), which reflects the property of surrounding tissues. In general, $\mathrm{ADC} D$ depends on $\mathbf{G}$ in a complex way, but free diffusion assumes that $D$ is only dependent on the direction of $\mathbf{G}$, i.e. $\mathbf{u}=\mathbf{G} / \mathbf{G}$. The early works in dMRI reported that the ADC $D$ is a scalar and independent of gradient direction $\mathbf{u}(102)$. Then, Dr. Basser introduced the diffusion tensor (14) to represent $\mathrm{ADC}$ as $D(\mathbf{u})=\mathbf{u}^{T} \mathbf{D u}$, where $\mathbf{D}$ is a $3 \quad 3$ symmetric positive definite matrix, called the diffusion tensor. This method is the well an known Diffusion Tensor Imaging (DTI). See Section 4.1.2 for more materials DTI.

The $b$ factor is given by $b=\gamma^{2} \delta^{2} \tau \mathbf{G}^{2}$, where $\gamma$ is the proton gyromagnetic ratio, and $\tau=\Delta \quad \delta / 3$ is used to describe the effective diffusion time $(25,14)$. The $b$ value is dependent on the sequence, and it is different in different kinds of lobes in the diffusion sequence (24, Chap. 9). The signal intensity at each voxel in DWI is dependent on both surrounding structures and given a weighted magnetic gradient (25). See Figure 4.2 for the DWI images $S(b)$ with different $b$ values and different gradient directions $\mathbf{u}$. It can be seen that the DWI images are very noisy, especially for large $b$ values.

\subsubsection{Restricted Diffusion}

Although we have obtained (4.2) for the measured diffusion signal attenuation for free diffusion, the diffusion of water molecules is hindered by surrounding tissues, especially in white matter. We derive the diffusion signal attenuation $E(\mathbf{G}, \Delta, \delta)=S(\mathbf{G}, \Delta, \delta) / S(0)$ under such restricted diffusion as follows, where $S(\mathbf{G}, \Delta, \delta)$ is the DWI signal associated with imaging parameters $(\mathbf{G}, \Delta, \delta)$. For each voxel in $\mathbf{x}$-space, let $\rho\left(\mathbf{R}_{0}\right)$ denote the spin 


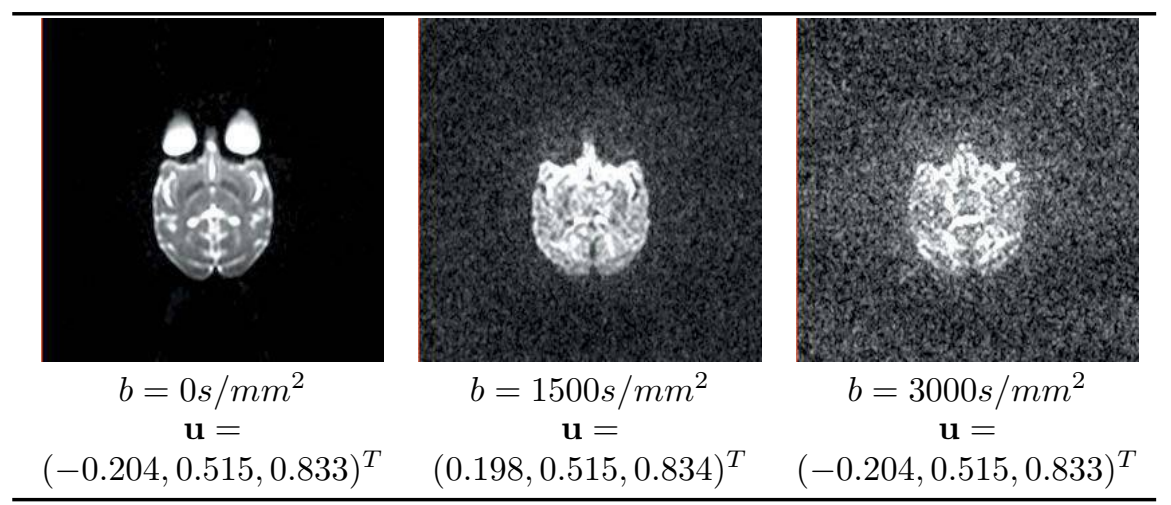

\section{FIGURE 4.2}

DWI images for different $b$ values and gradients. The data is from one of the subjects in a real monkey dataset.

density at initial time $t=0$ and $P\left(\mathbf{R}_{\Delta} \mathbf{R}_{0}\right)$ denote the probability that the spin moves from $\mathbf{R}_{0}$ at $t=0$ to $\mathbf{R}_{\Delta}$ at $t=\Delta$. Then $E(\mathbf{G}, \Delta, \delta)$ can be represented as (126)

$$
\int_{\mathbb{R}^{3}} \rho\left(\mathbf{R}_{0}\right) \int_{\mathbb{R}^{3}} P\left(\mathbf{R}_{\Delta} \mathbf{R}_{0}\right) \exp \left(i \gamma\left(\mathbf{R}_{\Delta} \quad \mathbf{R}_{0}\right)^{T}\left(\int_{0}^{\delta} \mathbf{G}(t) \mathrm{d} t\right)\right) \mathrm{d} \mathbf{R}_{\Delta} \mathrm{d} \mathbf{R}_{0} .
$$

We simplify $E(\mathbf{G}, \Delta, \delta)$ under the narrow pulse condition, that is, $0 \quad \delta<<\Delta$. We first define the Ensemble Average Propagator (EAP) as

$$
P(\mathbf{R})=\int_{\mathbb{R}^{3}} \rho\left(\mathbf{R}_{0}\right) P\left(\mathbf{R}_{0}+\mathbf{R} \mathbf{R}_{0}\right) \mathrm{d} \mathbf{R}_{0}, \quad \mathbf{R}=\mathbf{R}_{\Delta} \quad \mathbf{R}_{0}
$$

where $\mathbf{R}=\mathbf{R} \mathbf{r}$ is the displacement vector in $\mathbf{R}$-space and $\mathbf{r}$ is a unit vector. EAPs in different regions in the brain reflect the different micro-structures and reveal fiber directions. See Figure 4.3 for diffusion data in a six-dimensional space including i.e. 3D k-space and $3 \mathrm{D} \mathbf{q}$-space. Since $\mathbf{G}(t)$ is a constant during $\delta$, we may introduce a $\mathbf{q}$ vector in $\mathbf{q}$-space as

$$
\mathbf{q}=q \mathbf{u}=(2 \pi){ }^{1} \gamma \int_{0}^{\delta} \mathbf{G}(t) \mathrm{d} t=(2 \pi){ }^{1} \gamma \delta \mathbf{G} .
$$

Thus, $E(\mathbf{G}, \Delta, \delta)$ can be written as

$$
E(\mathbf{q})=\int_{\mathbb{R}^{3}} P(\mathbf{R}) \exp \left(2 \pi i \mathbf{q}^{T} \mathbf{R}\right) \mathrm{d} \mathbf{R}=\mathscr{F}_{3 D}^{1} P(\mathbf{R})(\mathbf{q}),
$$

where $\mathscr{F}_{3 D}$ and $\mathscr{F}_{3 D}^{1}$, respectively, denote the Fourier transformation and its inverse transformation. Without confusion, we call both $E(\mathbf{q})$ and $E(b)$ diffusion signals. Since $P(\mathbf{R})=P(\mathbf{R})$ holds due to the principle of microscopic detailed balance, we have $E(\mathbf{q})=E(\mathbf{q})=\mathscr{F} P(\mathbf{R})(\mathbf{q})$ leading to

$$
\int_{\mathbb{R}^{3}} P(\mathbf{R}) \exp \left(2 \pi i \mathbf{q}^{T} \mathbf{R}\right) \mathrm{d} \mathbf{R}=\int_{\mathbb{R}^{3}} P(\mathbf{R}) \cos \left(2 \pi \mathbf{q}^{T} \mathbf{R}\right) \mathrm{d} \mathbf{R} .
$$

Since the DWI data $S(\mathbf{q})$ is the Fourier transform of the $\mathbf{k}$-space signal and the EAP is another Fourier transform of $E(\mathbf{q})$, we can represent $P(\mathbf{R})$ as

$$
P(\mathbf{R})=\mathscr{F}_{3 D} E(\mathbf{q})(\mathbf{R})=\mathscr{F}_{3 D} \frac{S(\mathbf{G}, \Delta, \delta)}{S(0)}(\mathbf{R}) .
$$




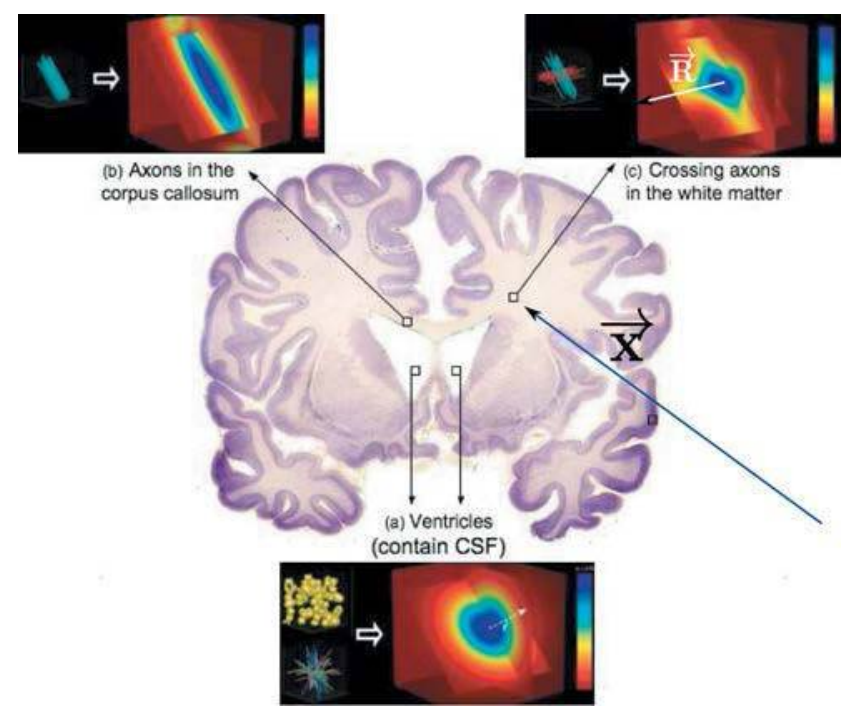

\section{FIGURE 4.3}

3D $\mathbf{x}$-space and 3D $\mathbf{R}$-space. EAPs in different regions in the brain reflect different micro-structures with isotropic diffusion, single fiber, and crossing fibers. The image is taken from (46) with the original figures adapted from (72) and the brain museum (www.brainmuseum.org/Specimens).

Due to $E(\mathbf{q})=E(\mathbf{q}), P(\mathbf{R})$ can be further written as

$$
\int_{\mathbb{R}^{3}} E(\mathbf{q}) \exp \left(2 \pi i \mathbf{q}^{T} \mathbf{R}\right) \mathrm{d} \mathbf{q}=\int_{\mathbb{R}^{3}} E(\mathbf{q}) \cos \left(2 \pi \mathbf{q}^{T} \mathbf{R}\right) \mathrm{d} \mathbf{q} .
$$

\subsubsection{Diffusion Tensor Imaging (DTI)}

In (14), Dr. Basser proposed to model the ADC as a quadratic form parameterized by the diffusion tensor D. Then the Stejskal-Tanner equation becomes

$$
E(b)=\exp \left(b \mathbf{u}^{T} \mathbf{D u}\right) .
$$

The diffusion tensor $\mathbf{D}=\sum_{k=1}^{3} \lambda_{k} \mathbf{e}_{k} \mathbf{e}_{k}^{T} \quad S y m_{3}^{+}$is independent of the $b$ value and gradient direction $\mathbf{u}$, where $\left(\lambda_{k}, \mathbf{e}_{k}\right)_{k}{ }_{3}$ are eigenvalue-eigenvector pairs such that $\lambda_{1} \lambda_{2} \quad \lambda_{3}$ and $\mathrm{Sym}_{3}^{+}$is the space of $3 \quad 3$ symmetric positive definite matrices. In free diffusion, $P(\mathbf{R})$ is given by

$$
P(\mathbf{R})=\mathscr{F}_{3 D} \exp \left(4 \pi^{2} \tau \mathbf{q}^{T} \mathbf{D q}\right)=\phi(\mathbf{R} \mathbf{0}, 2 \tau \mathbf{D}),
$$

where $\phi(\mathbf{R} \mathbf{0}, 2 \tau \mathbf{D})$ denotes the Gaussian density with mean $\mathbf{0}$ and covariance $2 \tau \mathbf{D}$. See Figure 4.4 for the sketch map of the tensor representation and free diffusion along fibers.

\subsubsection{Scalar Indices and Eigenvectors of Diffusion Tensor}

Several scalar indices based on $\mathbf{D}$ have been widely used in various biomedical studies. The two most important indices include Fractional Anisotropy (FA) and Mean Diffusivity 


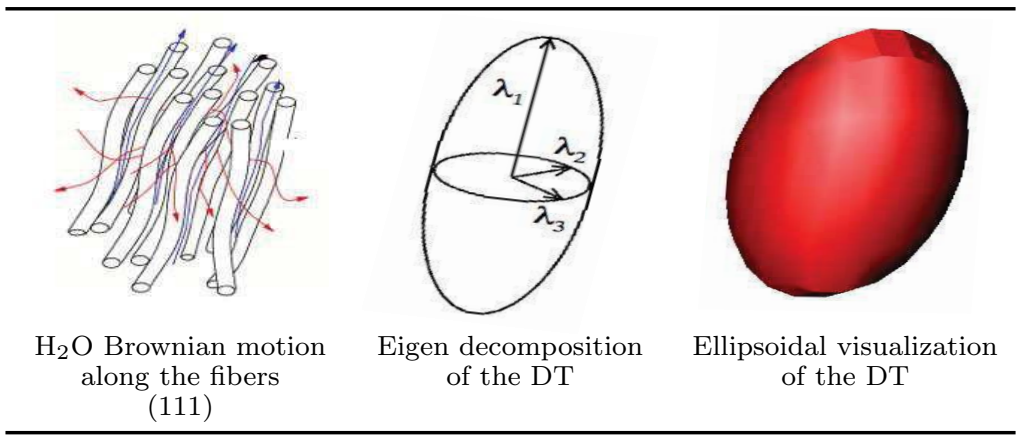

FIGURE 4.4

Diffusion tensor representation from (46).

(MD) (109) given by

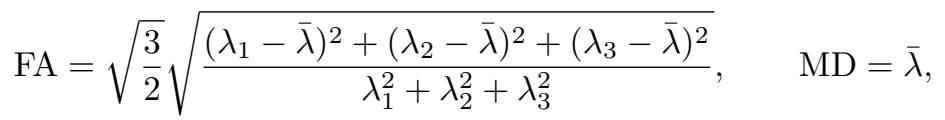

where $\bar{\lambda}=\left(\lambda_{1}+\lambda_{2}+\lambda_{3}\right) / 3$. In (143), linear, planar, and spherical measures are introduced as

$$
\mathrm{LA}=\left(\begin{array}{ll}
\lambda_{1} & \lambda_{2}
\end{array}\right) /(3 \bar{\lambda}), \quad \mathrm{PA}=2\left(\begin{array}{ll}
\lambda_{2} & \lambda_{3}
\end{array}\right) /(3 \bar{\lambda}), \quad \mathrm{SA}=\lambda_{3} / \bar{\lambda}
$$

The eigenvectors of $\mathbf{D}$ are also very useful. When $\lambda_{1}>\lambda_{2}$, the eigenvector corresponding to the largest eigenvalue is expected to be parallel to the local fiber orientation. In practice, the red-blue-green (RGB) map is used to describe the fiber directions. The tensor $\mathbf{D}$ itself can be visualized by a ellipsoid, then the tensor field becomes the ellipsoid field. See Figure 4.5 for the tensor field and various scalar maps estimated from an monkey data with $b=$ $1500 \mathrm{~s} / \mathrm{mm}^{2}, 30$ directions, where the Geodesic Anisotropy (GA) is introduced in (108).

\subsection{High Angular Resolution Diffusion Imaging (HARDI)}

The term High Angular Resolution Diffusion Imaging (HARDI) was first proposed by Tuch $(137,136)$ in order to have a more precise angular characterization of the diffusion signal. In this chapter, HARDI methods include all diffusion modeling methods beyond DTI. The HARDI methods for single shell (only one $b$ value) data are called sHARDI methods. The HARDI methods for multiple shell (multiple $b$ values) data are called mHARDI methods.

\subsubsection{Generalization of Diffusion Tensor Imaging}

\subsubsection{Mixture of Tensor Model}

The mixture of tensor model assumes that the signal is a mixture of signals generated from multiple tensors $\mathbf{D}_{k} \underset{k=1}{K}$ given by

$$
E(b)=\sum_{k}^{K} w_{k} \exp \left(b \mathbf{u}^{T} \mathbf{D}_{k} \mathbf{u}\right) \quad \text { with } \sum_{k=1}^{K} w_{k}=1 \text { and } w_{k} \quad 0 .
$$




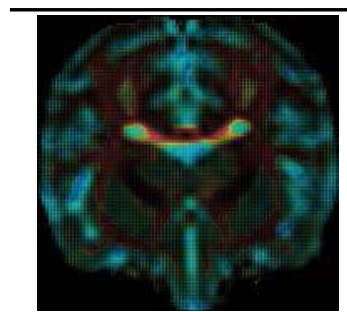

tensor field

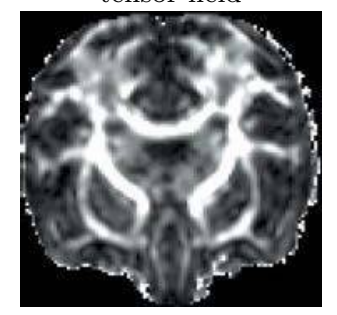

GA map

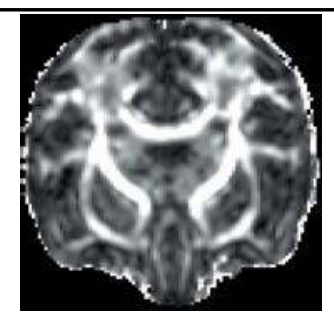

FA map

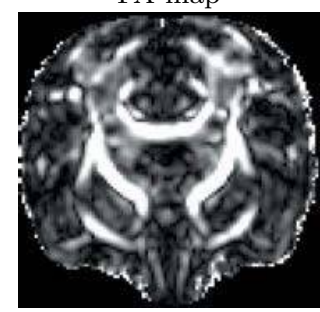

LA map

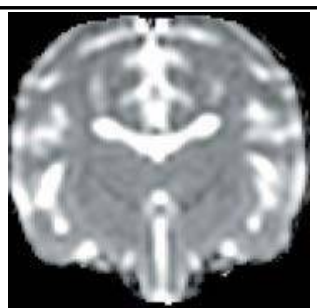

MD map

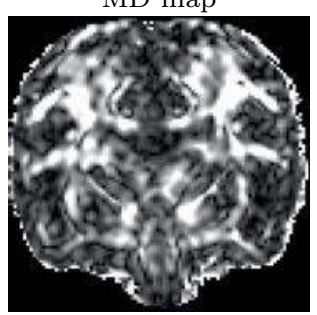

PA map

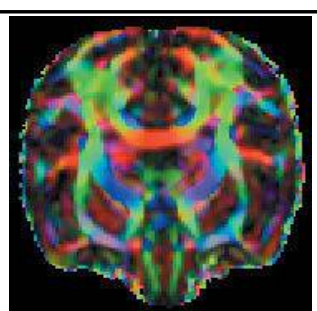

RGB map

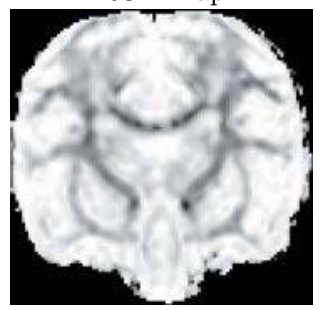

SA map

\section{FIGURE 4.5}

Tensor field and the scalar maps estimated from the monkey data with $b=1500 \mathrm{~s} / \mathrm{mm}^{2}$ and 30 directions.

Due to some biological priors and computational complexity, the number of tensors is normally less than 3 , typically $K=2$. Computationally, although various optimization algorithms may be developed to estimate $\mathbf{D}_{k} \mathrm{~s}$ (136), they may be computationally unstable and sensitive to the initial point. Moreover, for single shell data, the isotropic part of $\mathbf{D}_{i}$, i.e. Trace $\left(\mathbf{D}_{i}\right) / 3$, and $w_{i}$ are undistinguishable (87). Thus, some constraints on tensors are normally imposed in model (4.14). For example, the two minimal eigenvalues $\lambda_{2}$ and $\lambda_{3}$ in $\mathbf{D}_{i}$ can be chosen as the same value. Tensors $\mathbf{D}_{i}$ can be chosen as one isotropic tensor and other anisotropic tensor with $\lambda_{2}=\lambda_{3}=0$, which is called the ball and stick model (74). CHARMED model (10) considers $\mathbf{D}_{i}$ as a hindered diffusion part that is close to Gaussian diffusion, and a restricted diffusion part that is non-Gaussian diffusion. The mixture of tensor model has been widely used to generate synthetic data for evaluation because many quantities have closed forms in this model.

Model (4.14) has three major limitations. (i) Selecting the number of tensors is an open problem. (ii) The optimization process strongly depends on the initial point and is computationally inefficient. (iii) The radial decay of the mixture of tensor model is close to, but is not, the Gaussian function. Consider the number of tensors is $K=2$ in Figure 4.6; along a given direction, one component decays fast and the other one decays slowly. For large $b$ values, the component with slow decay dominates the signal.

\subsubsection{Generalized DTI (GDTI)}

In the GDTI model $(92,91)$, the signal is represented as

$$
E(\mathbf{q})=\exp \left(\sum_{l=2}^{L}(2 \pi i)^{l}\left(\Delta \quad \frac{l}{l+1} \delta\right) D_{i_{1} i_{2} \ldots i_{l}}^{(l)} q_{i_{1}} q_{i_{2}} \quad q_{i_{l}}\right),
$$

where we use the Einstein summation convention, i.e., $D_{i_{1} i_{2} \ldots i_{l}}^{(l)} q_{i_{1}} q_{i_{2}} \quad q_{i_{l}}=\sum_{i_{1}=1}^{3} \sum_{i_{2}=1}^{3}$ $\sum_{i_{l}=1}^{3} D_{i_{1} i_{2} \ldots i_{l}}^{(l)} q_{i_{1}} q_{i_{2}} \quad q_{i_{l}}$. When $L=2$, the GDTI becomes the DTI model in Eq. (4.10). 
The generalized diffusion coefficients $D_{i_{1} i_{2} \ldots i_{l}}^{(l)}$ can be estimated by the least square fitting the samples of $\ln E(\mathbf{q})$. Due to the relationship between $E(\mathbf{q})$ and $P(\mathbf{R})$ in (4.6), $E(\mathbf{q})$ is the characteristic function of $P(\mathbf{R})(91)$ given by

$$
E(\mathbf{q})=\exp \left(\sum_{l=0}^{L}(2 \pi i)^{l} Q_{i_{1} i_{2} \ldots i_{l}}^{(l)} q_{i_{1}} \quad q_{i_{l}} / l !\right)
$$

where $Q_{i_{1} i_{2} \ldots i_{l}}^{(l)}$ s are the cumulants. Then, based on the property of Hermite polynomial $H e_{n}(x)$, we can obtain the closed form for the EAP by using the Gram-Charlier A series (91), which leads to

$$
P(\mathbf{R})=N\left(\mathbf{R ~ 0}, Q_{i_{1} i_{2}}^{(2)}\right)\left(1+\sum_{l=3}^{L} \frac{Q_{i_{1} i_{2} \ldots i_{l}}^{(l)}}{l !} H e_{i_{1} i_{2} \ldots i_{l}}^{(l)}(\mathbf{R})\right),
$$

where $H e_{i_{1} i_{2} \ldots i_{l}}^{(l)}(\mathbf{R})$ is the $l$-order Hermite polynomial defined as

$$
(1)^{l} \exp \left(0.5 \mathbf{R}^{T} \mathbf{R}\right)\left(\begin{array}{cc}
\frac{\partial}{\partial R_{i_{1}}} \frac{\partial}{\partial R_{i_{2}}} & \frac{\partial}{\partial R_{i_{l}}}
\end{array}\right) \exp \left(0.5 \mathbf{R}^{T} \mathbf{R}\right) .
$$

There are several major limitations associated with the GDTI. It models the ADC using the polynomial basis, which is not orthogonal. Although theoretically the ADC can be modeled as infinite terms, in practice a truncated order $L$ is needed in Eq. (4.15). However, it was proved in (95) that the Gaussian distribution is the only distribution that has a finite number of non-zero cumulants. Thus a truncation order $L$ only results in a reasonable PDF if the EAP is Gaussian and $L=2$ in this case. For other cases, the estimated EAP and cumulants are theoretically problematic. Moreover, estimation of the PDF from its cumulants is known to be very problematic.

\subsubsection{High-Order Tensor Model, ADC-Based Model}

The High-Order Tensor (HOT) model (106) assumes

$$
E(\mathbf{q})=\exp \left(4 \pi^{2} \tau q^{2} D(\mathbf{u})\right)
$$

The ADC is independent of radial part $q$, and can be represented as

$$
D(\mathbf{u})=\sum_{n_{1}+n_{2}+n_{3}=L} D_{n_{1} n_{2} n_{3}} u_{1}^{n_{1}} u_{2}^{n_{2}} u_{3}^{n_{3}}
$$

where $\mathbf{u}=\left(u_{1}, u_{2}, u_{3}\right)^{T} \quad \mathbb{S}^{2}$, and $L$ is even due to $D(\mathbf{u})=D(\mathbf{u})$. Moreover, $u_{1}^{n_{1}} u_{2}^{n_{2}} u_{3}^{n_{3}} n_{1}+n_{2}+n_{3}=L$ is the homogeneous polynomial basis restricted in $\mathbb{S}^{2}$, which is also called the High-Order Tensor (HOT) basis in dMRI domain. When $L=2$, HOT model is just the DTI model in Eq. (4.10).

In the HOT, the diffusion signal decays as a mono-exponential function, which is called mono-exponential decay assumption, given by

$$
E(q \mathbf{u})=E\left(q_{0} \mathbf{u}\right)^{q^{2} / q_{0}^{2}}
$$

where $q_{0}$ and $q$ are any two different radii. Compared with the GDTI, which is model-free method, HOT is model-based. The mono-exponential decay assumption is not satisfied in real signal decay (88), but it can be a good approximation of the signal, especially when the $b$ value is around $1500 \mathrm{~s} / \mathrm{mm}^{2}(105)$. 


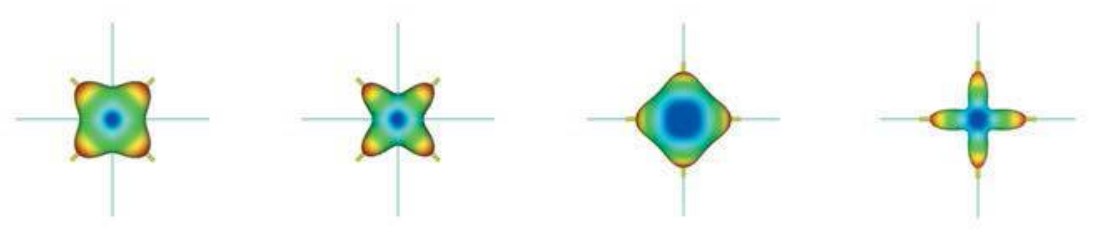

ADC $b=1500 s / m^{2}$ ADC $b=3000 s / m^{2}$ ODF by Tuch $\Phi_{t}(\mathbf{r})$ ODF by Wedeen $\Phi_{w}(\mathbf{r})$

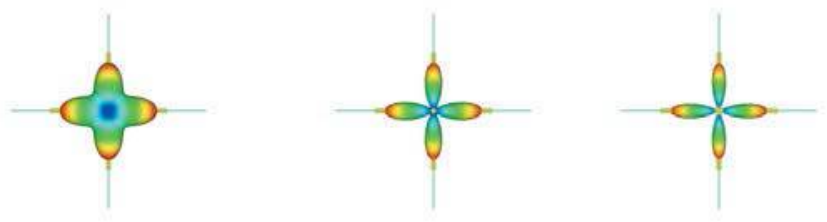

EAP profile with $R=10 \mu m \quad R=15 \mu m \quad R=20 \mu m$

\section{FIGURE 4.6}

Fiber directions and ADC profiles with different $b$ values, two kinds of ODFs, and EAP profiles with different radius $R$. The data was generated from the mixture of tensor model with two tensors that have the eigenvalues $[1.7,0.3,0.3] \quad 10^{3} \mathrm{~mm}^{2} / \mathrm{s}$ and a crossing angle of 90 . We set $\tau=\frac{1}{4 \pi^{2}}$ such that $b=q^{2}$. The long sticks with blue color along the $x$ - and $y$-axes are the fiber directions. The short sticks with yellow color are the detected maxima of the spherical functions.

The HOT model often uses single shell data in Figure 4.10(c), which is a kind of sHARDI method. Historically, people used both the High-Order Tensor basis (106) and Spherical Harmonic ( $\mathrm{SH}$ ) basis (62) to estimate ADC from a measured signal. Theoretically, these two bases are equivalent to each other. In HARDI literatures, the maximal order of the SH basis or the order of the HOT basis must be higher than 4, because the order 2 of the $\mathrm{SH}$ basis and HOT basis are equivalent to the tensor model. Normally 4 or 6 is used in practice.

ADC modeling, like the HOT method, has its intrinsic and fatal limitation, i.e. both the maxima and the minima of ADC profile $D(\mathbf{u})$ are inconsistent with the fiber directions when $L>2$ (139). Figure 4.6 demonstrates the ADC $D(\mathbf{u})$ for the synthetic data generated from the mixture of tensor model with a crossing angle of 90 . It shows that the maxima of ADC do not agree with the fiber directions. Even in this simple mixture of tensor model, the ADC $D$ is actually dependent on the $b$ value, and the mono-exponential decay assumption is violated. For the data with different $b$ values, the $\mathrm{ADC}$ is determined by $D=b^{1} \ln E(\mathbf{q})$, which means $D$ is dependent on $b$ if $E(\mathbf{q})=\sum_{i=1}^{K} \exp \left(b \mathbf{u}^{T} \mathbf{D}_{i} \mathbf{u}\right)$. Although there is a coincidence that the minima of ADC agree with the fiber directions in this specific case of the mixture of tensor model with 90 , the minima and maxima of the ADC generally have a complex relation with fiber directions.

\subsubsection{Diffusion Spectrum Imaging (DSI)}

Due to Eq. (4.9) for the narrow pulse assumption $\delta \quad \Delta$, a straightforward idea is to estimate $P(\mathbf{R})$ using fast Fourier transform from exhaustive signal samples (142). For instance, (142) used 515 DWI images in a Cartesian sampling lattice in $\mathbf{q}$-space and the signal in 


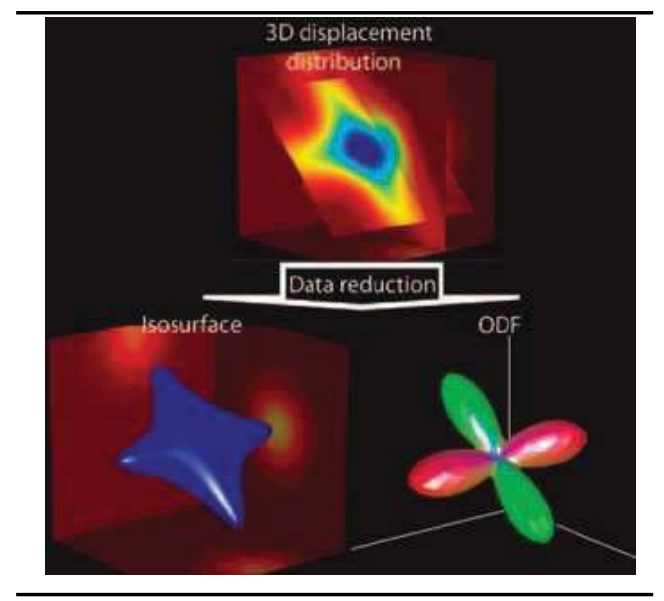

\section{FIGURE 4.7}

EAP in 3D R-space and its two features, i.e. EAP profile (or called iso-surface of EAP) and ODF. The figure is from (72).

q-space was premultiplied by a Hanning window to obtain smooth attenuation of the signal at high $\mathbf{q}$ values. Thus interpolation and extrapolation are normally performed on given signal samples $E\left(\mathbf{q}_{i}\right)$ before numerical Fourier transform.

In (142), the EAP profile is EAP at a given radius $R_{0}$ as

$$
P\left(R_{0} \mathbf{r}\right)=P(R \mathbf{r})_{R=R_{0}} .
$$

The maxima of the EAP profile were used to describe fiber directions later in many HARDI works $(105,11,48)$. See Figure 4.6 for the EAP profile with different radius $R$. The larger the radius $R$, the sharper the EAP profile is. However, the EAP profile with large $R$ has more estimation error. Thus normally $R=15 \mu \mathrm{m}$ is used in the EAP profile to detect the fiber directions $(105,48)$.

In (142), the ODF is defined as

$$
\Phi_{w}(\mathbf{r}) \stackrel{\text { def }}{=} \int_{0} P(\mathbf{R}) R^{2} \mathrm{~d} R .
$$

Since $\Phi_{w}(\mathbf{r})$ is the marginal distribution of $\operatorname{EAP} P(\mathbf{R})$, the integration of $\Phi_{w}(\mathbf{r})$ over $\mathbb{S}^{2}$ is naturally 1. Reference (142) proposed to first estimate the EAP via numerical Fourier transform and then estimate the ODF in Eq. (4.22) by numerical integration. Historically there are several kinds of ODFs which can be seen in the following of this section. Like the EAP profile, the maxima of ODFs are also normally assumed to be the directions of underlying fibers. Please see Figure 4.6 for the EAP in 3D space and its two features, i.e. the EAP profile and ODF.

\subsubsection{Hybrid Diffusion Imaging (HYDI)}

Hybrid Diffusion Imaging (HYDI) uses multiple shell sampling to measures the diffusion signal in q-space $(147,148)$. See Figure $4.10(\mathrm{~d})$. The HYDI data in the shell with low $b$ values can be modeled by DTI, whereas those data with high $b$ values can be modeled by using Q-Ball Imaging and other sHARDI methods. The whole HYDI dataset can be used in DSI after re-griding data from multiple shells to the Cartesian lattice. 
Two useful scalar features of EAP in HYDI include the Return-to-Origin probability (RTO) and the Mean Squared Displacement (MSD). The RTO denoted by $P_{o}$ is the EAP value when $R=0$, that is, $P_{o}=P(\mathbf{R})_{R=0}=P(0)=\int_{\mathbb{R}^{3}} E(\mathbf{q})$ dq. The RTO is the probability of water molecules that minimally diffuse within the diffusion time $\Delta$. The RTO map can be used in tissue segmentation and some other applications (147). The MSD is the variance of the EAP, that is, $M S D=\int_{\mathbb{R}^{3}} P(\mathbf{R}) \mathbf{R}^{T} \mathbf{R} \mathrm{d} \mathbf{R}$. In (148), the ODF by Tuch $\Phi_{t}(\mathbf{r})$ in Eq. (4.24) is proportional to the integration of $E(\mathbf{q})$ in the orthogonal plane

$$
\Pi_{\mathbf{r}}=q \mathbf{u}: \mathbf{u}^{T} \mathbf{r}=0
$$

It is an important relation between the ODF by Tuch in $\mathbf{R}$-space and the signal $E(\mathbf{q})$ in $\mathbf{q}-$ space, and it is used in exact QBI to estimate ODFs analytically $(1,133)$. See Section 4.2 .4 for more details on QBI and ODFs.

\subsubsection{Q-Ball Imaging (QBI)}

Q-Ball Imaging (QBI) is currently the most widely used HARDI method. QBI was proposed to estimate the several kinds of ODFs, not the EAP, from single shell sampling demonstrated in Figure 4.10(c), rather than Cartesian sampling inside a given ball used in DSI in 4.10(b).

\subsubsection{Original Q-Ball Imaging}

QBI was first proposed by Dr. Tuch in $(135,134)$ in a numerical way and then was improved in an analytical way based on the Spherical Harmonic basis in $(7,47)$. Dr. Tuch originally proposed to estimate a kind of ODF defined as

$$
\Phi_{t}(\mathbf{r}) \stackrel{\text { def }}{=} \frac{1}{Z} \int_{0} P(R \mathbf{r}) \mathrm{d} R
$$

where $Z$ is the normalization factor that makes $\int_{\mathbb{S}^{2}} \Phi_{t}(\mathbf{r}) \mathrm{d} \mathbf{r}=1$. This $\Phi_{t}(\mathbf{r})$ is different from the ODF $\Phi_{w}(\mathbf{r})$ defined in Eq. (4.22).

In QBI, Dr. Tuch proposed to estimate $\Phi_{t}(\mathbf{r})$ directly from samples of $E(\mathbf{q})$ in singleshell data based on the Funk-Radon transform (FRT). See Figure 4.10(c) for the sketch map of single shell sampling. For single-shell data with $b=4 \pi^{2} \tau q_{0}^{2}$, the FRT of $E(\mathbf{q})$ (134) in direction $\mathbf{r}$, denoted as FRT $E\left(q_{0} \mathbf{u}\right)(\mathbf{r})$, is the circle integration in the orthogonal plane, i.e.

$$
\int_{\Pi_{\mathbf{r}}} E(q \mathbf{u}) \delta\left(q \quad q_{0}\right) q \mathrm{~d} q \mathrm{~d} \mathbf{u}=q_{0} \int_{\mathbf{u} \mathbb{S}^{2}} E\left(q_{0} \mathbf{u}\right) \delta\left(\mathbf{u}^{T} \mathbf{r}\right) \mathrm{d} \mathbf{u},
$$

where $\Pi_{\mathbf{r}}$ is defined in Eq. (4.23). The ODF $\Phi_{t}(\mathbf{r})$ can be written as

$$
\begin{aligned}
& (2 Z)^{1} \iint_{0}^{2 \pi} \int_{0} P\left(r_{\mathbf{R}}, \phi_{\mathbf{R}}, z_{\mathbf{R}}\right) \delta\left(r_{\mathbf{R}}\right) \delta\left(\phi_{\mathbf{R}}\right) r_{\mathbf{R}} \mathrm{d} r_{\mathbf{R}} \mathrm{d} \theta_{\mathbf{R}} \mathrm{d} z_{\mathbf{R}} \\
& 2 \pi q_{0} Z^{1} \iint_{0}^{2 \pi} \int_{0} P\left(r_{\mathbf{R}}, \phi_{\mathbf{R}}, z_{\mathbf{R}}\right) J_{0}\left(2 \pi q_{0} r_{\mathbf{R}}\right) r_{\mathbf{R}} \mathrm{d} r_{\mathbf{R}} \mathrm{d} \theta_{\mathbf{R}} \mathrm{d} z_{\mathbf{R}}
\end{aligned}
$$

which equals $Z{ }^{1} \mathrm{FRT} E\left(q_{0} \mathbf{u}\right)(\mathbf{r})$, where $J_{0}()$ is the Bessel function of the first kind with order 0. The key idea of Eq. (4.26) is to approximate the delta function using Bessel function $0.5 a J_{0}(a x)$. As $q_{0}$ increases, $2 \pi q_{0} J_{0}\left(2 \pi q_{0} r_{\mathbf{R}}\right)$ will be very close to the delta function, since the lobes of $J_{0}$ become more concentrated around the origin point. However, the signal has smaller values for larger $q_{0}$, which results in a low signal-to-noise ratio (SNR). Thus there is a tradeoff for $q_{0}$ between approximation accuracy and SNR. Normally QBI works suggest data with $b$ values around $3000 \mathrm{~s} / \mathrm{mm}^{2}(134,47)$. 
Dr. Tuch proposed to estimate the circle integration in FRT using numerical integration (134). The numerical QBI was later replaced by analytical QBI based on $E\left(q_{0} \mathbf{u}\right)=$ $\sum_{l=0}^{L} \sum_{m=l}^{l} c_{l m} Y_{l}^{m}(\mathbf{u})$, where $Y_{l}^{m}(\mathbf{u})$ is the symmetric real spherical harmonic with order $l$ and degree $m$ (7). We have

$$
\widetilde{\Phi_{t}}(\mathbf{r})=Z^{1} \mathrm{FRT} E\left(q_{0} \mathbf{u}\right)(\mathbf{r})=Z^{1} \sum_{l=0}^{L} \sum_{m=l}^{l} 2 \pi P_{l}(0) c_{l m} Y_{l}^{m}(\mathbf{u})
$$

where $P_{l}(0)$ is the Legendre polynomial of order $l$ evaluated at 0 . In practice the ODF by Tuch $\Phi_{t}(\mathbf{r})$ in Eq. (4.24) is very smooth. The peaks of the ODF are only a little higher than the baseline values. Dr. Tuch proposed a min-max normalization method for visualization of $\Phi_{t}(\mathbf{r})$ to enhance the peaks of ODFs. However, the min-max normalization also enhances the peaks of the ODFs in the area with isotropic diffusion. Compared with ADC-based modeling like the HOT method, the maxima of ODFs agree with the fiber directions. Dr. Tuch also proposed a useful scalar index, named Generalized Fractional Anisotropy (GFA), to describe the anisotropy of the ODFs given by

$$
\text { GFA } \Phi_{t}(\mathbf{r}) \stackrel{\text { def }}{=} \sqrt{\frac{N \sum_{i=1}^{N}\left(\Phi_{t}\left(\mathbf{r}_{i}\right) \Phi_{t}(\mathbf{r})\right)^{2}}{\left(n \text { 1) } \sum_{i=1}^{N} \Phi_{t}\left(\mathbf{r}_{i}\right)^{2}\right.}},
$$

where $\Phi_{t}(\mathbf{r})$ is the mean of $\Phi_{t}(\mathbf{r})$.

QBI has several major limitations. (i) The ODF by Wedeen $\Phi_{w}(\mathbf{r})$ defined in Eq. (4.22) is theoretically sharper than $\Phi_{t}(\mathbf{r})$. (ii) The estimation via FRT has an intrinsic blurring effect, which leads to smoothed ODFs. (iii) QBI actually assumes the radial part of $E(\mathbf{q})$ as a delta function, which is unrealistic. The burring effect from FRT is the direct consequence from this assumption of radial decay. (iv) QBI cannot be used in multiple-shell data, because the data from different $b$ values leads to different ODFs from FRT.

\subsubsection{Exact Q-Ball Imaging}

Exact QBI was proposed by several groups independently $(148,133)$ to estimate ODFs through the famous projection-slice theorem in Fourier transform ${ }^{2}$ : the projection of $P(R \mathbf{r})$ along direction $\mathbf{r}$, i.e. the radial integration, equals the integration of $E(\mathbf{q})$ in the orthogonal plane $\Pi_{\mathbf{r}}$. Thus we have the following corollary, which is a straightforward result of the above proposition and has been used to estimate both ODF by Tuch and ODF by Wedeen $(148,1,131,133,39)$. The $\Phi_{t}(\mathbf{r})$ and $\Phi_{w}(\mathbf{r})$ can be written as

$$
\Phi_{t}(\mathbf{r})=Z^{1} \int_{\Pi_{\mathbf{r}}} E(\mathbf{q}) \mathrm{d} \mathbf{q}, \quad \Phi_{w}(\mathbf{r})=(4 \pi)^{1} \quad\left(8 \pi^{2}\right){ }^{1} \int_{\Pi_{\mathbf{r}}} q^{1} \Delta_{b} E(\mathbf{q}) \mathrm{d} \mathbf{q},
$$

where $\Delta_{b}$ is the Laplace-Beltrami operator in $\mathbb{S}^{2}$. If $E(\mathbf{q})$ follows the mono-exponential decay assumption (1), then we have

$$
\Phi_{w}(\mathbf{r})=\frac{1}{4 \pi}+\frac{1}{16 \pi^{2}} \int_{\mathbb{S}^{2}} \Delta_{b} \ln \left(\ln E\left(q_{0} \mathbf{u}\right)\right) \delta\left(\mathbf{u}^{T} \mathbf{r}\right) \mathrm{d} \mathbf{u} .
$$

By representing $\ln \left(\ln E\left(q_{0} \mathbf{u}\right)\right)=\sum_{l=0}^{L} \sum_{m=l}^{l} c_{l m} Y_{l}^{m}(\mathbf{u})$, and considering $\Delta_{b} Y_{l}^{m}(\mathbf{u})=$ $l(l+1) Y_{l}^{m}(\mathbf{u})$, we have $\Phi_{w}(\mathbf{r})=\frac{1}{4 \pi} \quad \frac{1}{8 \pi} l(l+1) P_{l}(0) c_{l m} Y_{l}^{m}(\mathbf{u})$. Although the monoexponential decay assumption is better than the delta function assumption, it is still a strong and unrealistic assumption of radial decay; even the signal generalized by a simple mixture of tensor model does not follow this assumption as shown in Figure 4.6. Exact QBI is a kind of sHARDI method and leads to different results for the data from different shells.

\footnotetext{
${ }^{2}$ http://en.wikipedia.org/wiki/Projection-slice_theorem
} 


\subsubsection{Diffusion Orientation Transform (DOT)}

Diffusion Orientation Transform (DOT) was proposed by Dr. Özarslan in (105) to estimate the EAP profile $P(R \mathbf{r})$ from single-shell data under the mono-exponential decay assumption in Eq. (4.18). It can be regarded as an estimator of EAP in exact QBI methods. Consider the plane wave equation as

$$
\cos \left(2 \pi \mathbf{q}^{T} \mathbf{R}\right)=4 \pi \sum_{l=0} \sum_{m=l}^{l}(1)^{l / 2} j_{l}(2 \pi q R) Y_{l}^{m}(\mathbf{u}) Y_{l}^{m}(\mathbf{r}),
$$

where $j_{l}($ ) is the $l$-order spherical Bessel function. Thus, we have

$$
P(R \mathbf{r})=\sum_{l=0} \sum_{m=l}^{l}\left(\int_{\mathbb{S}^{2}} Y_{l}^{m}(\mathbf{u}) I_{l}(R, \mathbf{u}) \mathrm{d} \mathbf{u}\right) Y_{l}^{m}(\mathbf{r}),
$$

where $I_{l}(R, \mathbf{u})=4 \pi(\quad 1)^{l / 2} \int_{0} E(\mathbf{q}) j_{l}(2 \pi q R) q^{2} d q$ can be calculated analytically based on the samples of ADC $D\left(\mathbf{u}_{j}\right)$. Then a least square fitting can be used to obtain the coefficients of $P\left(R_{0} \mathbf{r}\right)$ under the $\mathrm{SH}$ basis from $I_{l}\left(R_{0}, \mathbf{u}_{j}\right)$.

Reference (105) validated the mono-exponential decay assumption through synthetic data generated from the cylinder model. It showed that signal decay can be approximated well as a mono-exponential function around $b=1500 \mathrm{~s} / \mathrm{mm}^{2}$. For the $b$ value larger than $3000 \mathrm{~s} / \mathrm{mm}^{2}$, the mono-exponential decay assumption is not well satisfied, and the data with large $b$ value has low SNR. Thus $1500 \mathrm{~s} / \mathrm{mm}^{2}$ seems to be the optimal $b$ value for DOT. Note like original QBI and exact QBI, DOT cannot handle multiple-shell data, since the data in different shells leads to different EAP profiles.

\subsubsection{Spherical Deconvolution (SD)}

Spherical Deconvolution (SD) methods $(130,129)$ generalize the mixture model from the discrete case to the continuous case by assuming

$$
E(q \mathbf{u})=\int_{\mathbb{S}^{2}} \Phi_{f}(\mathbf{r}) R\left(\mathbf{r}^{T} \mathbf{u}\right) \mathrm{d} \mathbf{r},
$$

where $\Phi_{f}(\mathbf{r})$ is the fiber ODF (fODF) and $R\left(\mathbf{r}^{T} \mathbf{u}\right)$ is the fiber response signal generated from one fiber. The spherical deconvolution is a model-based method because it assumes the typical signal $R\left(\mathbf{r}^{T} \mathbf{u}\right)$ and linear combination in the convolution. The mixture of tensor model suffers from the model selection of the number of tensors and local minima of cost function. In contrast, SD can be solved analytically by considering the closed-form expression of spherical integration using the $\mathrm{SH}$ basis representation of the $E(q \mathbf{u})$ and $R\left(\mathbf{r}^{T} \mathbf{u}\right)$ using SHs $(129,49)$. SD can be used for DWI signal $E(q \mathbf{u})$ or ODFs/EAPs calculated from DWI signal (49). However the direct usage of SD for the DWI signal is theoretically better because the estimation of ODFs/EAPs from DWI signal always suffers from noise and insufficient samples. SD methods normally obtain many false positive fODF peaks when the signal is less anisotropic. The false positive peaks can be largely reduced by considering the non-negativity constraint of the estimated fODFs $(129,38)$. Conventional SD is only for single-shell data. However by introducing a 3-dimensional fiber response function, SD methods can be used in multi-shell data (38).

\subsubsection{Diffusion Propagator Imaging (DPI)}

Diffusion Propagator Imaging (DPI) was proposed to model the signal $E(\mathbf{q})$ as the solution of Laplace's equation (48). DPI can be seen as a generalization of the QBI method to handle 
multiple-shell data, although this generalization has many problems. In DPI, the signal is assumed to be

$$
E(q \mathbf{u})=\sum_{l=0}^{L} \sum_{m=l}^{l}\left(\frac{c_{l m}}{q^{l+1}}+d_{l m} q^{l}\right) Y_{l}^{m}(\mathbf{u}) .
$$

Then, the EAP is estimated from incomplete 3D integration inside the ball with a given radius $q_{\max }$, which is the maximum $q$ value used in DPI acquisition, since the complete integration in $\mathbb{R}^{3}$ does not converge. After obtaining the coefficients $c_{l m}$ and $d_{l m}$, several EAP features can be calculated analytically based on incomplete radial integration. However, DPI has some limitations. (i) The estimated ODFs suffer from two incomplete integrations including one for EAP estimation and the other for ODF estimation. (ii) It is unclear how to choose $q_{\max }$. (iii) The DPI model does not satisfy some priors of signal $E(\mathbf{q})$, which brings intrinsic modeling errors. It also cannot represent an isotropic Gaussian signal.

\subsubsection{Simple Harmonic Oscillator Reconstruction and Estimation (SHORE)}

SHORE was proposed by Dr. Özarslan in (104) for 3D signals. In SHORE, $E(\mathbf{q})$ in 3D (41) is represented by

$$
E(\mathbf{q})=\sum_{n=0}^{N} \sum_{l=0}^{2 n} \sum_{m=l}^{l} a_{n l m} B_{n l m}^{\mathrm{SHO} 3}(\mathbf{q} \zeta)
$$

where $B_{n l m}^{\mathrm{SHO} 3}(\mathbf{q} \zeta)=G_{n l}(q \zeta) Y_{l}^{m}(\mathbf{u})$ and $G_{n l}(q \zeta)$ is a given function and depends on $l$. SHORE is model-free, since the SHO basis is a complete basis in $\mathbb{R}^{3}$. The linear analytical solutions are very fast and avoid numerical integration. However, the representation in (4.34) is not non-negative in nature. After estimating $a_{n l m}$, the EAP can be analytically calculated as

$$
P(\mathbf{R})=\sum_{n=0}^{N} \sum_{l=0}^{2 n} \sum_{m=l}^{l} a_{n l m}(1)^{n} G_{n l}\left(R \frac{1}{4 \pi^{2} \zeta}\right) Y_{l}^{m}(\mathbf{r}) .
$$

The two kinds of ODFs can also be analytically derived from the estimated coefficients.

\subsubsection{Spherical Polar Fourier Imaging (SPFI)}

Spherical Polar Fourier Imaging (SPFI) was first proposed by Dr. Assemlal in (11) in a numerical way and then improved by $(40,39)$. SPFI represents the diffusion signal $E(\mathbf{q})$ as a linear combination of the SPF basis, i.e.,

$$
E(\mathbf{q})=\sum_{n=0}^{N} \sum_{l=0}^{L} \sum_{m=l}^{l} a_{n l m} B_{n l m}^{\mathrm{SPF}}(\mathbf{q} \zeta)
$$

where $B_{n l m}^{\mathrm{SPF}}(\mathbf{q} \zeta)=G_{n}(q \zeta) Y_{l}^{m}(\mathbf{u})$, in which $\kappa_{n}(\zeta)^{2}=2 n ! /\left[\zeta^{3 / 2} \Gamma(n+3 / 2)\right]$ and $G_{n}(q \zeta)=$ $\kappa_{n}(\zeta) \exp \left(q^{2} /(2 \zeta)\right) L_{n}^{1 / 2}\left(q^{2} / \zeta\right)$. The SPF basis is a 3D orthonormal basis with SHs in the spherical part and Gaussian Laguerre functions in the radial part. SPFI is model-free, since the SPF basis is complete in their domains. The linear analytical solutions are very fast and avoid numerical integration. However, the representation Eq. (4.36) is not non-negative in nature. After we estimate the coefficients of the diffusion signal under SPF basis, the EAP and its various features, e.g., ODFs, RTO, can be obtained in an analytical way $(40,39)$. 


\subsection{Reconstruction}

DMRI data consists of $n$ DWIs with $n$ measurements $\left(S\left(\mathbf{q}_{i} ; \mathbf{v}\right), \mathbf{r}_{i}, b_{i}\right): i=1, \quad, n$ at voxel $\mathbf{v}$ in a common space $\mathcal{V}$. A reconstruction step in dMRI is to estimate ODF $\Phi_{w}(\mathbf{r} ; \mathbf{v})$, and $\operatorname{EAP} p(\mathbf{R} ; \mathbf{v})$ at each voxel $\mathbf{v} \quad \mathcal{V}$. To design an efficient method to accurately reconstruct the ODF and EAP, one must address three key components of dMRI data including (i) the model for the diffusion signal attenuation; (ii) the noise components in dMRI data; and (iiii) the spatial/functional nature of dMRI data. Since various models for $E(\mathbf{q} ; \mathbf{v})$ have been extensively reviewed above, we focus on the last two key components here. If such models for $E(\mathbf{q} ; \mathbf{v})$ were misspecified, one would not expect to accurately reconstruct the ODF and/or EAP. We will discuss why the last two components of dMRI data are critical for dMRI reconstruction.

\subsubsection{Noise Components and Voxelwise Estimation Methods}

DWIs inherently contain varying amounts of noise that must be modeled or corrected appropriately if ODFs and EAPs are to be estimated accurately; failure to do so may lead to a biased estimate of the ODF (or EAP) and to incorrect estimates of their associated invariant measures (e.g., GFA). The measured diffusion weighted signals, however, can contain varying amounts of noise of diverse origins, including noise from stochastic variation, numerous physiological processes, eddy currents, artifacts from the differing magnetic field susceptibilities of neighboring tissues, rigid body motion, nonrigid motion, and many others $(82,83)$. Some noise components, such as bulk motion from cardiac pulsation and head or body movement, generate unusual observations, or statistical outliers. Previous studies have shown that those noise components can introduce substantial bias into measurements and estimations made from those images, such as invariant measures and fiber tracts in diffusion tensor images $(152,96,35)$. Identifying and reducing these noise components in DWIs is essential to improving the validity and accuracy of DTI studies designed to map brain structure and function.

Two types of approaches, including various robust statistical methods and diagnostic methods, have been proposed to address the 'non-random' noise components in DWIs. Robust statistical methods produce the estimators that are insensitive to significant deviations from the model assumption, while incorporating the properties of classic statistics $(76,43)$. Specifically, in DTI, several robust approaches have been used to exclude outliers from the diffusion signal attenuation in order to improve the accuracy of tensor estimation $(34,35,96)$. These proposed algorithms, however, only work properly for a small number of outliers in the case of high SNR. Diagnostic methods based on some influence measures (e.g., Cook's distance) can isolate outliers caused by certain noise components, including motion artifacts (167). An adaptive estimation procedure can be used to refit to dMRI data in order to obtain refined estimators by downweighting outliers.

In the presence of random noise only, the signal intensity in DWIs acquired from a single coil follows a Rician distribution, denoted by $S\left(\mathbf{q}_{i} ; \mathbf{v}\right) \quad R\left(\mu_{i}\left(\mathbf{q}_{i}, \mathbf{v}\right), \sigma^{2}(v)\right)(122)$. As shown (167), a general Rician regression model was introduced and an expectation-maximization (EM) algorithm was first proposed to calculate the maximum likelihood estimate of unknown parameters. Moreover, the Rician distribution can be well approximated by a normal distribution $N\left(\sqrt{\mu_{i}\left(\mathbf{q}_{i}, \mathbf{v}\right)^{2}+\sigma(\mathbf{v})^{2}}, \sigma(\mathbf{v})^{2}\right)$ when SNR 2 and $N\left(\mu_{i}\left(\mathbf{q}_{i}, \mathbf{v}\right), \sigma(\mathbf{v})^{2}\right)$ when SNR 5. The log-transformed signal intensity $\log S\left(\mathbf{q}_{i} ; \mathbf{v}\right)$ approximately follows a weighted Gaussian distribution $N\left(\log \left(\mu_{i}\left(\mathbf{q}_{i}, \mathbf{v}\right)\right), \sigma^{2}(v) / \mu_{i}\left(\mathbf{q}_{i}, \mathbf{v}\right)^{2}\right)(13,6,165)$. For DTI in (4.10), we have $\log \left(\mu_{i}\left(\mathbf{q}_{i}, \mathbf{v}\right)\right)=\log S(0) \quad b \mathbf{u}^{T} \mathbf{D u}$. An efficient weighted least-square algorithm was 
developed to reconstruct $\mathbf{D}(165)$. If DWIs are acquired from multiple coils, $S\left(\mathbf{q}_{i} ; \mathbf{v}\right)$ is non-central Chi (nc- $\chi$ ) distributed, provided that the $k$ space is fully sampled and no correlations between the coil data exists (2). Recently, the estimation method under the nc- $\chi$ noise has been developed to estimate $\mathbf{D}$ in (23).

Raw HARDI images, as a result of elevated $b$ factor and decreased voxel size, suffer from depressed SNRs, which make the problem of reconstructing HARDI data of practical importance and challenging. Most HARDI reconstruction algorithms directly assume that

$$
f\left(E\left(\mathbf{q}_{i} ; \mathbf{v}\right)\right)=\mathbf{x}_{i}^{T} \beta(\mathbf{v})+\epsilon_{i}(\mathbf{v}),
$$

where $f()$ is a given transformation function (e.g., $f(s)=s$ or $f(s)=\log (s))$, $\mathbf{x}_{i}$ is a $p$ vector of covariates, which depends on $\mathbf{q}_{i}$ (or $\left(\mathbf{b}_{i}, r_{i}\right)$ ), $\beta(\mathbf{v})$ is a $p \quad 1$ vector of regression coefficients, and $\epsilon_{i}(\mathbf{v})$ is an error term with mean zero and variance $\sigma_{i}^{2}(\mathbf{v})$. Model (4.37) is general enough to cover many existing HARDIs. In the literature, for GDTI and HOT, it is common to set $f\left(E\left(\mathbf{q}_{i} ; \mathbf{v}\right)\right)=\log \left(E\left(\mathbf{q}_{i} ; \mathbf{v}\right)\right)$ and represent $\log \left(E\left(\mathbf{q}_{i} ; \mathbf{v}\right)\right)$ as a polynomial function of $\mathbf{q}_{i}$, whereas for most other HARDIs, such as QBI or DOT, it is common to set $f\left(E\left(\mathbf{q}_{i} ; \mathbf{v}\right)\right)=E\left(\mathbf{q}_{i} ; \mathbf{v}\right)$ and approximate $E\left(\mathbf{q}_{i} ; \mathbf{v}\right)$ by a linear combination of some basis functions, such as the spherical polar Fourier basis.

Most HARDI methods focus on the reconstruction of $\beta(\mathbf{v})$ by solving a regularized linear least-squares optimization problem

$$
\widehat{\beta}(\mathbf{v})=\operatorname{argmin}_{\beta(\mathbf{v})} \mathbf{y}(\mathbf{v}) \quad \mathbf{X} \beta(\mathbf{v})^{2}+\rho(\beta(\mathbf{v}) ; \lambda(\mathbf{v})),
$$

where $\mathbf{y}(\mathbf{v})=\left(f\left(E\left(\mathbf{q}_{1} ; \mathbf{v}\right)\right), \quad, f\left(E\left(\mathbf{q}_{n} ; \mathbf{v}\right)\right)\right)^{T}, \mathbf{X}$ is an $n \quad p$ matrix with the $i$-th row being $\mathbf{x}_{i}$, and $\rho(\beta(\mathbf{v}) ; \lambda(\mathbf{v}))$ is a penalty function with $\lambda(\mathbf{v})$ being a tuning parameter. Different penalty functions, such as LASSO and the Laplacian-Beltrami, can be used in (4.38) $(128,47,98)$. Recently, as discussed in (98) and references therein, there is a high interest in developing the compression sensing technique for dMRI applications. In Bayesian statistics, $\quad \log (\rho(\beta(\mathbf{v}) ; \lambda(\mathbf{v})))$ can be regarded as the prior of $\beta(\mathbf{v})$.

Existing methods based on (4.37) and (4.38) have at least three major limitations. First, these methods largely ignore the stochastic noise components of the DW signal that we discussed above. Therefore, it may lead to bias and loss of efficiency in the estimation of the ODF and EAP. Second, these methods perform reconstruction independently at each voxel, which essentially ignores the functional nature of the DWI data at different voxels in space. Third, most HARDI reconstruction algorithms often use some heuristic methods to determine a single value of $\lambda(\mathbf{v})$ for all voxels. However, both theoretically and numerically, the selection of the tuning parameter across voxels plays a critical role in ensuring the nice properties of the regularized estimators (28).

\subsubsection{Spatial-Adaptive Estimation Methods}

Recently, there is a growing interest in developing spatial-adaptive estimation methods for the HARDI/DTI reconstruction in order to characterize the spatial/functional nature of DWIs. Until recently, a number of different approaches have been developed starting from smoothing raw DWIs $(50,19,18,115,20,21)$, smoothing procedures in tensor space $(99,60)$, smoothing procedures in ODF space $(85,68)$, spatial DTI $(127,155,154,93)$, to spatial HARDI, which reconstructs and denoises all ODFs simultaneously (113). The key idea of these methods is to explicitly incorporate spatial smoothness constraints into various HARDI reconstruction algorithms. The key assumption of this type of approach is that the orientation and anisotropy of any single fiber population are expected to vary smoothly along the dominant fiber orientation, except at the boundaries between tracts and interfaces with gray matter structures and cerebrospinal fluid spaces. Mathematically, DWI 
can be characterized as a convolution of a piecewise smooth function with various MRI noises.

Most spatial-adaptive methods can be classified into three categories including (i) the denoising of raw DWI data, (ii) the denoising of the estimated tensor/EAP/ODF field, and (iii) simultaneous smoothing and estimation of DWI data. In the first category, most approaches to DWI denoising are designed to incorporate the stochastic components of raw DWI data with their spatial smoothness by using either regularization methods or nonparametric statistical methods. The DWI denoising method is to denoise the observed $\left(S\left(\mathbf{q}_{i} ; \mathbf{v}\right): \mathbf{v} \quad \mathcal{V}\right)_{i} 1$ in order to calculate the denoised DWIs, denoted by $\left(S\left(\mathbf{q}_{i} ; \mathbf{v}\right)\right.$ : $\mathbf{v} \mathcal{V})_{i} 1_{1}$. The regularization-based denoising methods estimate $S\left(\mathbf{q}_{i} ; \mathbf{v}\right)$ by solving a regularized optimization problem

$$
\operatorname{argmin}_{S_{*}\left(\mathbf{q}_{i} ; \mathbf{v}\right)} \int_{\mathbf{v} \mathcal{V}} \ell\left(S\left(\mathbf{q}_{i} ; \mathbf{v}\right), S\left(\mathbf{q}_{i} ; \mathbf{v}\right)\right)+\rho\left(S\left(\mathbf{q}_{i} ; \mathbf{v}\right), \lambda(\mathbf{v})\right) d L(\mathbf{v}),
$$

where $\quad \ell\left(S\left(\mathbf{q}_{i} ; \mathbf{v}\right), S\left(\mathbf{q}_{i} ; \mathbf{v}\right)\right)$ is usually chosen to be the log-likelihood function of the DW signal at voxel $\mathbf{v}, \rho($,$) is a pre-specified penalty function, such as total variation, and L(\mathbf{v})$ is a measure defined on $\mathcal{V}$. Various penalty functions include those associated with total variation schemes, Markov random fields, and Perona-Malik-like smoothing. In Bayesian statistics, $\log \left(\rho\left(S\left(\mathbf{q}_{i} ; \mathbf{v}\right), \lambda(\mathbf{v})\right)\right)$ is the prior of $S\left(\mathbf{q}_{i} ; \mathbf{v}\right)$ in the $(\mathbf{q}, \mathbf{v})$ space.

Nonparametric statistical methods incorporate both spatial proximity and similarity measure to calculate weighted averages of 'similar' DW signals in order to explicitly account for the piecewisely smooth nature of imaging data with jumps and edges. These similar signals can be incorporated in denoising from both the spatial $\mathbf{v}$-space and the diffusion q-space. Some well-known methods include non-local means (NLM) and unbiased NLM algorithms, propagation-separation methods, anisotropic Wiener filtering, the bilateral filter, and the Sigma filter, among others $(110,8,151,27,84)$. For instance, NLM uses small sub-images, called patches, to denoise the image by accounting for the redundancy in natural images, especially in textured parts $(8,27,84)$. Based on the Rician noise, a NLM for DWI data can be formulated as

$$
\operatorname{NLM}(S(\mathbf{q}, \mathbf{v}))=\sqrt{\sum_{\left(\mathbf{q}^{\prime}, \mathbf{v}^{\prime}\right) V_{(\mathbf{q}, \mathbf{v})}} w((\mathbf{q}, \mathbf{v}),(\mathbf{q}, \mathbf{v})) S(\mathbf{q}, \mathbf{v})^{2} 2 \sigma^{2}},
$$

where $w((\mathbf{q}, \mathbf{v}),(\mathbf{q}, \mathbf{v}))$ is defined by the distance of the patches centered in $(\mathbf{q}, \mathbf{v})$ and $(\mathbf{q}, \mathbf{v})$, and $\sigma$ is a global noise variance. The NLM can be performed separately for different $\mathbf{q}$ or jointly for all signals in $\mathbf{q}$ space by defining $w($,$) based on vector-valued patches$ $(51,145)$. When the noise variance is unknown, it can be estimated jointly from all signals in q space via a linear minimum mean square error (LMMSE) estimator $(3,132)$. However, NLM has some limitations for piecewise smooth images when the noise is not small (8). This is exactly the case for DWI. In contrast, the propagation-separation (PS) method is very efficient at smoothing noisy piecewise smooth images and dealing with edges (18), even though such methods cannot proceed efficiently in textured regions. The key idea of the PS method is to construct a sequence of nested local neighborhoods (or patches) adapted to DW signals in its neighboring voxels and then adaptively estimate $S\left(\mathbf{q}_{i} ; \mathbf{v}\right)$ at each voxel. Although the PS method is computationally extensive, it is robust to the selection of kernel window sizes and patch shapes at different locations.

In the second category, most methods perform denoising on the estimation tensor/ODF/EAP results by using either regularization methods or nonparametric statistical methods $(97,108,99,68,162,157,61,121,119,54,37)$. For DTI, various regularizationbased denoising methods estimate a tensor field $\mathbf{D}(\mathbf{v}): \mathbf{v} \quad \mathcal{V}$ based on the estimated 
tensor field $\widehat{\mathbf{D}}(\mathbf{v}): \mathbf{v} \quad \mathcal{V} \quad(36,45)$. Mathematically, it can be formulated as

$$
\operatorname{argmin} \mathbf{D}(\mathbf{v}): \mathbf{v} \mathcal{V} \int_{\mathbf{v}} \mathcal{V} d(\widehat{\mathbf{D}}(\mathbf{v}), \mathbf{D}(\mathbf{v}))+\rho(\mathbf{D}(\mathbf{v}), \lambda(\mathbf{v})) d L(\mathbf{v}),
$$

where $d(\widehat{\mathbf{D}}(\mathbf{v}), \mathbf{D}(\mathbf{v}))$ is usually chosen to be a pre-specified distance between $\widehat{\mathbf{D}}(\mathbf{v})$ and $\mathbf{D}(\mathbf{v})$ and $\rho($,$) is a pre-specified penalty function on the tensor field. Similar to (4.39),$ various penalty functions can be developed for the tensor field based on total variation schemes, Markov random fields, and Perona-Malik-like smoothing. Since D lies in a curved space, one has to face additional theoretical and computational challenges.

For DTI, nonparametric statistical methods have been developed to estimate an intrinsic 'expectation' (or 'median') of a symmetric positive definite matrix response $\mathbf{D}$, given a voxel location $\mathbf{v}$ from a set of estimated diffusion tensors $\left(\mathbf{v}_{1}, \widehat{\mathbf{D}}\left(\mathbf{v}_{1}\right)\right), \ldots,\left(\mathbf{v}_{m}, \widehat{\mathbf{D}}\left(\mathbf{v}_{m}\right)\right)$, in which $\mathbf{v}$ may belong to the set of $\mathbf{v}_{1}, \quad, \mathbf{v}_{m}$. Mathematically, it can be formulated by solving a weighted estimator of $\mathbf{D}(\mathbf{v})$ that is defined by

$$
\tilde{\mathbf{D}}^{\alpha}(\mathbf{v})=\operatorname{argmin}_{\mathbf{D}(\mathbf{v})} \sum_{i=1}^{m} w\left(\mathbf{v}_{m}, \mathbf{v}\right) g\left(\widehat{\mathbf{D}}_{m}\left(\mathbf{v}_{m}\right), \mathbf{D}(\mathbf{v})\right)^{\alpha},
$$

where $\alpha \quad 1, w(\mathbf{v}, \mathbf{v})$ is defined by the weighted 'distance' of voxels $\mathbf{v}$ and $\mathbf{v}$, and $g(\mathbf{D}(\mathbf{v}), \mathbf{D}(\mathbf{v}))$ is the geodesic distance between $\mathbf{D}(\mathbf{v})$ and $\mathbf{D}(\mathbf{v})$. Moreover, $\tilde{\mathbf{D}}^{1}(\mathbf{v})$ and $\tilde{\mathbf{D}}^{2}(\mathbf{v})$ are, respectively, an intrinsic median estimator and an intrinsic least square estimator of $\mathbf{D}(\mathbf{v})(61,60,157,30)$. Two commonly used metrics, including the trace metric and the log-Euclidean metric, are usually chosen for the geodesic distance on the space of symmetric positive definite matrices $(9,157,54)$. Directly solving $(4.42)$ is equivalent to the calculation of an intrinsic local constant estimator of $\mathbf{D}(\mathbf{v})$. In (157), the authors propose a general intrinsic local polynomial regression estimate for the analysis of symmetric positive definite matrices as responses. For each metric, they develop a cross-validation bandwidth selection method, derive the asymptotic bias, variance, and normality of the intrinsic local constant and local linear estimators, and compare their asymptotic mean-square errors. For the ODF, $(55,68)$ develop an intrinsic local constant estimator of the ODF in order to smooth ODF imaging, but its related statistical theory has not been established yet.

In the third category, a few methods have been developed to perform simultaneous smoothing and estimation of DTI by using either regularization methods or nonparametric statistical methods $(127,154,93)$. Specifically, (93) proposed to solve a regularized optimization problem

$$
\begin{aligned}
& \underset{\mathbf{a r g m i n}}{\operatorname{D}(\mathbf{v}): \mathbf{v}} \mathcal{V} \int_{\mathbf{v}} \ell_{n}\left(S\left(\mathbf{q}_{i} ; \mathbf{v}\right) i_{i} ; \mathbf{D}(\mathbf{v})\right) d L(\mathbf{v}) \\
& +\lambda_{1} \int_{\mathbf{v}} \int_{W(\mathbf{v})} \omega(\mathbf{v}, \mathbf{v}) g(\mathbf{D}(\mathbf{v}), \mathbf{D}(\mathbf{v})) d \mathbf{v} d \mathbf{v}
\end{aligned}
$$

where $\lambda_{1}$ is a tuning parameter, $\ell_{n}\left(S\left(\mathbf{q}_{i} ; \mathbf{v}\right)_{i}{ }_{1} ; \mathbf{D}(\mathbf{v})\right)$ is usually chosen to be the loglikelihood function of the observed DW signals $S\left(\mathbf{q}_{i} ; \mathbf{v}\right)_{i}{ }_{1}, \omega(\mathbf{v}, \mathbf{v})$ are the regularization weights, and $W(\mathbf{v})$ is the search window at voxel $\mathbf{v}$. Reference (93) proposed to use the weighting function of NLM to construct $\omega(\mathbf{v}, \mathbf{v})$ and use the total Bregman divergence to design $g(\mathbf{D}(\mathbf{v}), \mathbf{D}(\mathbf{v}))$.

A multiscale adaptive regression modelling (MARM) framework based on the PS method can be used to carry out simultaneous smoothing and estimation of DTI/ODF/EAP (90, 127). Specifically, let $B\left(\mathbf{v}, h_{s}\right)=\begin{array}{lllllll}\mathbf{v} & \mathbf{v} & \mathbf{v} & 2 & h_{s} & \text { be a sequence of balls centered at } \mathbf{v}\end{array}$ with increasing radii $h_{s}$ such that $h_{0}=0<h_{1}<\quad<h_{S}=r_{0}$. At each voxel $\mathbf{v}$, MARM 
iteratively maximizes a weighted objective function as

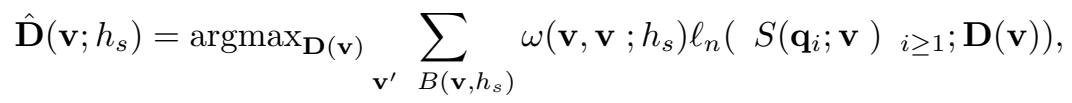

where $\omega\left(\mathbf{v}, \mathbf{v} ; h_{s}\right)$ s are adaptive weights calculated at each radius $h_{s}$ and allow us to incorporate data from neighboring voxels $\mathbf{v} \quad B\left(\mathbf{v}, h_{s}\right)$. At each voxel $\mathbf{v}$, we will obtain a sequence of estimators of $\mathbf{D}(\mathbf{v})$ as follows:

$$
\hat{\mathbf{D}}\left(\mathbf{v} ; h_{0}\right) \quad \omega\left(\mathbf{v}, \mathbf{v} ; h_{1}\right) \quad \omega\left(\mathbf{v}, \mathbf{v} ; h_{S}\right) \quad \hat{\mathbf{D}}\left(\mathbf{v} ; h_{S}\right) .
$$

When $s=0, \hat{\mathbf{D}}\left(\mathbf{v} ; h_{0}\right)$ reduces to the estimator of $\hat{\mathbf{D}}(\mathbf{v})$ for the voxel-wise method. Compared with the regularization method in (4.43), MARM should be more robust to higher noise levels and the selection of kernel window sizes and patch shapes at different locations. Finally, the adaptive weights in MARM can be extended to include the weighting function of NLM and/or existing biological information (e.g., fiber tracks). See Figure 4.8 (a)-(c) for an illustration of the use of MARM for ODF reconstruction based on QBI.

However, methods for each category have some advantages and disadvantages. (i) For the first category, these methods have been criticized for ignoring the fact that raw diffusion weighted signals acquired at different q-values are highly associated with each other in each voxel. Moreover, in white matter regions, the SNRs vary dramatically across different q values. Since these methods primarily use different weights to smooth the raw diffusion weighted images independently, such methods are prone to accumulate biases from all DWIs, which can lead to large biases in the estimated tensor/ODF/EAP images.

(ii) For the second category, these methods have been criticized for ignoring the stochastic components of the raw DWI data and directly smoothing the estimated tensor/ODF/EAP based on a specific metric of the tensor/ODF/EAP space. Since each estimated DTI is estimated by using all diffusion signals in each voxel, the estimated tensors can be shown to be asymptotically normal distributed with zero mean by using the central limit theorem. The estimated tensors are asymptotically unbiased and thus it is not critical to model the distribution of the stochastic components of the raw DWI data. Moreover, a key advantage of these methods is to use the same set of weights to smooth the raw DWI data across all q-values. However, if the originally estimated tensor/ODF/EAP field is biased, then these methods may not be able to reduce the biases in the smoothed tensor/ODF/EAP field.

(iii) For the third category, these methods are computationally more expensive, but a key advantage of these methods is to adaptively determine the weights at each voxel and then apply them to the raw DW signals. They avoid the potential biases introduced by those methods for the first category. Moreover, since MARM refits the raw DWI data at each bandwidth, it avoids the potential biases introduced by the voxelwise method.

\subsection{Tractography Algorithms}

Many tractography algorithms have been proposed to map fibers through the entire brain based on the estimated principal direction/ODF field $(59,116,77,89,100)$. The algorithms can be categorized into two main groups: local and global methods. Local methods use local ODF information to independently construct fibers path by path. Local methods can be grouped into two classes: deterministic and probabilistic. Deterministic algorithms usually start at seed voxels and follow the local principal directions/ODFs estimated by the diffusion 


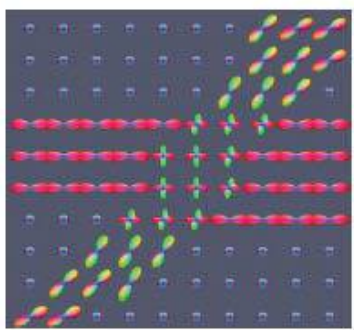

noise-free

(a)

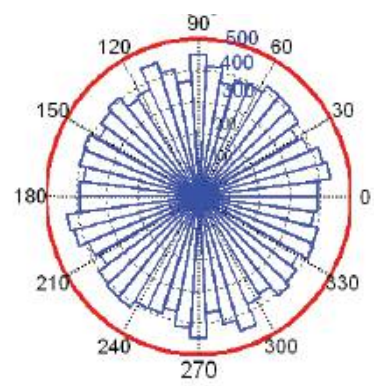

(d)

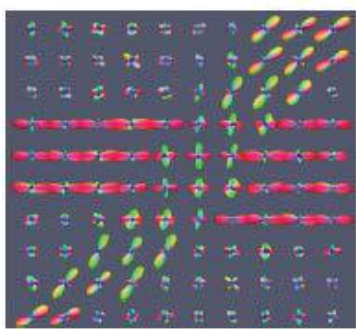

cQBI

(b)

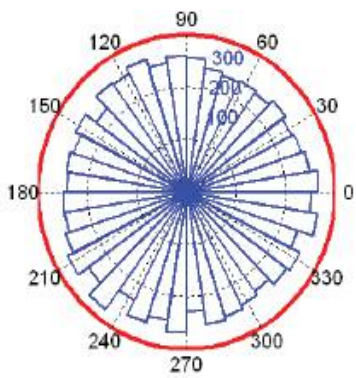

(e)

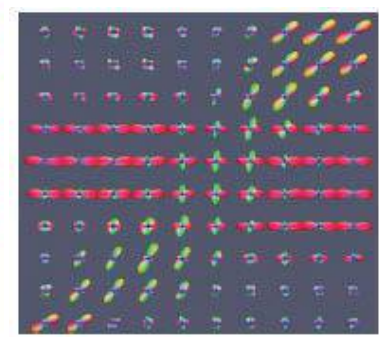

p-cQBI

(c)

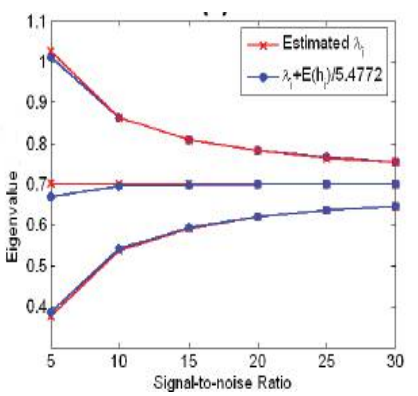

(f)

\section{FIGURE 4.8}

Simulation Results. The first row shows ODF reconstruction results based on simulated data with twisted crossing: true ODF field (a), estimated ODF fields based on cQBI (b), and MARM-cQBI (c). The second row shows results from a simulation study of the isotropic tensor $\mathbf{D}=\operatorname{diag}(0.7,0.7,0.7)$ (units: $\left.10{ }^{3} \mathrm{~mm}^{2} / s\right)$ : the angle histogram plots of $\theta$ based on 10,000 simulated DW datasets at $\mathrm{SNR}=20(\mathrm{~d})$ and 10,000 eigenvectors (e) simulated from the theoretical distribution given in (165), respectively, where $\theta \quad[0,2 \pi]$ is the subcomponent of $(1, \theta, \phi)$, the spherical coordinate of the eigenvector corresponding to the largest eigenvalue. (f) shows the theoretical means of the estimated three eigenvalues and the mean value of estimated eigenvalues as a function of SNR from 5 to 30 based on 10,000 simulated datasets.

model in order to generate sequences of points that are considered on major fibers. Several deterministic tractography algorithms include streamline algorithms and more elaborated tensor deflection algorithms, among others. Probabilistic algorithms repeatedly use Monte Carlo simulations (e.g., Markov chain Monte Carlo) to statistically generate the principal directions and then apply some deterministic methods for tracking fiber bundles. Such methods produce maps of 'probability' for each voxel to be crossed by a random track and the probabilistic maps of connectivity between any two ROIs. See $(78,141,89)$ for a nice review of various tractography algorithms and references therein. An advantage of local methods is their computational efficiency. However, the local methods can be very sensitive to noise components in DWIs, which can significantly affect the final tracking result.

Figure 4.9 showed fiber tracts across several ROIs by the deterministic local tractography method in MRtrix (See Section 4.9.2). The subject is from the Q3 dataset in the Human Connectome Project (HCP), where $b=1000,2000,3000 \mathrm{~s} / \mathrm{mm}^{2}, 90$ directions per shell. Constrained SD (129) was performed for all $90 x 3$ volumes using a 3D fiber response function (38) to estimate fODFs. Then MRtrix is used for deterministic local tractography. 


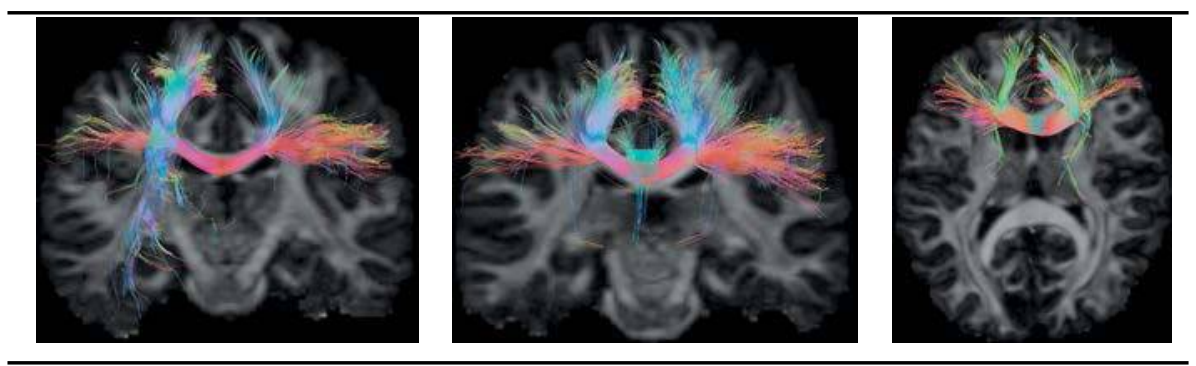

\section{FIGURE 4.9}

Fiber tracts of a subject in HCP Q3 dataset by a deterministic local tractography used in MRtrix.

We consider a stochastic differential equation model with measurement errors for local tractography methods $(86,118)$. Specifically, let $\mathbf{v}(t)$ be the true fiber trajectory in $R^{3}$. The stochastic differential equation model assumes

$$
\frac{d \mathbf{v}(t)}{d t}=\mathbf{e}(\mathbf{v}), \quad t \quad 0 \quad \text { with } \quad \mathbf{v}(0)=\mathbf{v}_{0}
$$

where $\mathbf{e}(\mathbf{v})$ is the true fiber direction at location $\mathbf{v}$ and $\mathbf{v}_{0}$ is the position of the seed location. Based on dMRI data, one is able to obtain an estimate of the true fiber direction field, denoted by $\hat{\mathbf{e}}(\mathbf{v}): \mathbf{v} \quad \mathcal{V}$, such that

$$
\hat{\mathbf{e}}(\mathbf{v})=\mathbf{e}(\mathbf{v})+\epsilon(\mathbf{v})
$$

where $\epsilon(\mathbf{v})$ is a zero-mean stochastic process. Numerically, let $\delta>0$ be a fixed approximation step and a sequence of points $t_{k}=k \delta$ for $k=0,1, \ldots,[T / \delta]$. By using Euler's approximation, one can solve (4.45) by iteratively updating

$$
\mathbf{v}\left(t_{k}\right)=\mathbf{v}\left(\begin{array}{ll}
t_{k} & 1
\end{array}\right)+\hat{\mathbf{e}}\left(\mathbf{v}\left(\begin{array}{ll}
t_{k} & 1
\end{array}\right)\right) \quad \text { for } \quad k=1, \quad,[T / \delta], \quad \mathbf{v}\left(t_{0}\right)=\mathbf{v}_{0} .
$$

Global methods reconstruct all detectable fibers of the brain simultaneously. It reconstruct fibers by finding a configuration that best describes the whole set of measured data (94). The reconstructed fibers are built by small line elements, each of them reflecting a part of the whole diffusion anisotropy. Elements being connected in lines eventually form reconstructed fibers. An advantage of global methods is stable with respect to noise and imaging artifacts. However, the global methods are often computationally time-consuming.

We consider a Bayesian approach for the global methods as follows. Let $\mathcal{M}$ be the assumed fiber model in $\mathcal{V} \quad R^{3}$ and $\mathcal{S}=\left(S\left(\mathbf{q}_{i} ; \mathbf{v}\right), \mathbf{q}_{i}\right): i=1, \quad, n$ be observed DWI data. One needs to specify a sampling distribution of $\mathcal{S}$ given $\mathcal{M}$, denoted by $p(\mathcal{S} \mathcal{M})$, and a prior distribution of $\mathcal{M}, p(\mathcal{M})$. For the sampling distribution, one can use the dMRI models discussed above. The key idea and challenge of the global tracking methods lies in how to specify the prior of the fiber model, $p(\mathcal{M})$. In $(116)$, the authors used small line (fiber) segments $L_{S}(\mathbf{v})=(\mathbf{v}, \mathbf{r}(\mathbf{v}))$ consisting of a continuous spatial position $\mathbf{v} \quad \mathcal{V}$ and an orientation $\mathbf{r}(\mathbf{v})$ that can form chains to represent the individual fibers. A mixture model of the product of a stick model in orientation space and an isotropic Gaussian in the spatial domain is assumed for $p(\mathcal{S} \mathcal{M})$. A simple interaction model is assumed for all connected segments, which leads to $p(\mathcal{M})$. The simulated annealing algorithm is used to calculate the posterior mode of $\hat{\mathcal{M}}=\operatorname{argmax}_{\mathcal{M}} p(\mathcal{M} \mathcal{S})$, where $p(\mathcal{M} \mathcal{S}) \quad p(\mathcal{S} \mathcal{M}) p(\mathcal{M})$ is the posterior distribution of $\mathcal{M}$ given $\mathcal{S}$. 
Despite the increasing availability of different tractography algorithms, there are many open questions in the quantification of these fiber-tracking methods.

It is critical to develop a reliable evaluation and validation system for tractography algorithms (44). The diffusion community has developed several evaluation measures (e.g., connectivity analysis) and two well-known phantoms including the FiberCup phantom dataset and the HARDI reconstruction challenge phantom to evaluate various diffusion models and tractography algorithms (59). An important finding is that probabilistic tractography algorithms lead to many false positives and should be used with caution (44). Much more research should be done on the design of evaluation measures and more realistic phantoms that are close to human brain in various settings.

Development of computationally efficient global tractography algorithms needs more attention. It is critical to develop a more reasonable fiber model $p(\mathcal{M})$ in order to better estimate the true fiber tracts and quantify their uncertainties. Moreover, optimizing $p(\mathcal{M S})$ is computationally challenging due to the presence of a large number of parameters and their non-convexity.

\subsection{Uncertainty in Estimated Diffusion Quantities}

Because of the noise that is inherent in DWI data, calculated tensors/ODFs/EAPs and their associated quantities (e.g., eigenvalues and principal directions) generally differ from the true ones, producing uncertainty in their estimation. To establish dMRI as a reliable and widely accepted technique, it is critical to quantify such uncertainty in various estimated diffusion quantities. Such quantification is very important for addressing many scientific questions in neuroscience and for designing and carrying out large DWI-related clinical studies.

Two classes of methods, including Monte Carlo and theoretical methods, have been developed to quantify estimated diffusion tensors and their eigenspace components. The Monte Carlo methods consist of (i) a statistical model for diffusion weighted signals; (ii) the choice of an estimation method; and (iii) the quantification of uncertainty in estimated diffusion quantities based on Monte Carlo simulations. In contrast, besides (i) and (ii), the theoretical methods use some mathematical and statistical techniques to directly approximate the uncertainty of estimated diffusion quantities instead of using Monte Carlo simulations.

Recent theoretical calculations based on perturbation theory and asymptotic theory have accurately approximated the uncertainty of the estimated eigenvalues and eigenvectors, as well as the bias that is introduced by sorting by their magnitudes eigenvalues in both degenerate and nondegenerate tensors $(6,165)$. Those calculations have shown in particular that the uncertainty in identifying a tensor's principal direction is determined primarily by whether the overall morphology of the tensor is degenerate or not (165). The results in the asymptotic theory allow us to delineate the stochastic behavior of estimated eigenvalues and eigenvectors for degenerate tensors, whereas those in the perturbation theory cannot. See Figure 4.8 (d)-(f) for an illustration of theoretical results for the isotropic tensor.

The Monte Carlo methods include both simulation studies and bootstrapping methods. Based on (4.10) and the Rician noise model, previous simulation studies have shown that estimated eigenvalues are always distinct and that their estimated FA is always greater than zero, regardless of whether the tensor is degenerate (i.e., oblate, prolate, or isotropic) or nondegenerate $(109,16,22)$. Thus, one always incorrectly identifies the principal directions of tensors within regions that contain isotropic or oblate tensors in real DWI. 
Bootstrapping methods, including repetition and the wild bootstrap, have been widely used to numerically quantify the uncertainty of eigenvalues, eigenvectors, and diffusion properties (144). Repetition bootstrap in DTI requires repeated measurements in each gradient direction, because it resamples with replacement the raw DW images in each of those directions. The accuracy of the repetition bootstrap depends on the number of repeated measurements in each direction. The wild bootstrap is a model-based method that resamples the residuals of the linear regression model used to estimate the tensor at each voxel. In particular, it is applicable to most DTI acquisition schemes, including the standard acquisition of one measurement per direction, unlike the repetition bootstrap (144).

One has to use the wild and repetition bootstrap methods with extra caution, since these methods have been used in the dMRI literature without any theoretical justification. However, such justification is necessary for producing any scientifically meaningful measure of diffusion uncertainty in which we are interested. In (158), the authors examine several fundamental issues associated with the two bootstrap methods by using both theoretical arguments and extensive Monte Carlo simulations. The two bootstrap methods are invalid for quantifying the uncertainty of the parameters for some tensors, such as the principal direction of an isotropic or a degenerate tensor. The validity of the wild bootstrap strongly depends on the correct specification of the fitted model used to estimate a tensor. Because the wild bootstrap resamples the residuals of the fitted tensor model, resampled tensors may not reflect the true characteristics of DTs in real DWIs.

There are many open questions in the quantification of the uncertainty in various estimated diffusion quantities.

For HARDI, little has been done to quantify the uncertainty of estimated ODFs and EAPs and their associated quantities based on the voxelwise estimation methods. Moreover, if one uses more complex spatial-adaptive estimation methods, such as PS, to estimate the ODF and EAP, such quantification becomes more difficult due to spatial smoothness and the presence of spatial correlation. According to the best of our knowledge, nothing has ever been done on such quantification.

How to quantify the uncertainty of estimated fiber tracks is largely unknown. Although there are a few attempts at quantifying of uncertainty in estimated tractography from both numerical and theoretical perspectives $(63,22,86)$, several critical issues remain open and need further theoretical investigation. Theoretically, (86) first proved some asymptotic/stochastic properties of the estimated tractography based on models (4.45)-(4.46). More research should be done along this direction. In contrast, although some existing DTI packages produce some uncertainty measures in the tractography results, it is unclear whether such measures are valid from a methodological perspective. For instance, one approach is to calculate the probability that two regions are connected based on local tractography algorithms and Monte Carlo methods, such as bootstrap. However, such probability may be positively correlated with the true probability that the two regions are connected, but they are not the same. Such probability should be used with great caution.

\subsection{Sampling Mechanisms}

An important design issue is how to select a set of gradients and $b$ values $\left(\mathbf{r}_{i}, b_{i}\right): i=$ $1, \ldots, n$ or $q$ values $\mathbf{q}_{i}: i=1, \ldots, n$ in order to accurately estimate tensor/ODF/EAP 
across all voxels. Statistically, this is an optimal design problem (12). Different sampling schemes in q-space have been developed in the literature (Figure 4.10). See (32) for an extensive review of various acquisition strategies in $q$ space. There are three principles for comparing different acquisition strategies including antipodal symmetry, being isotropic, and reconstruction. When there is no prior in the underlying tensor/ODF/EAP field, the first two principles have motivated people to uniformly arrange points on the sphere with central symmetry. Based on the third principle, various acquisition schemes have been developed to optimize tensor reconstruction $(64,107,123)$.

To select an efficient set of $q$ values, one must address three key components of dMRI data including (i) a statistical model for diffusion weighted signals; (ii) the choice of an appropriate optimality criterion, denoted by $L\left(\begin{array}{lll}\mathbf{q}_{i} & i & n\end{array}\right)$; and (iiii) optimizing the optimality criterion with respect to $\mathbf{q}_{i}: i=1, \ldots, n$. For (i), various models for dMRI signals have been developed above. For (ii), the optimality criterion is usually developed to quantify the uncertainty of the objective of interest, such as tensors and fiber tracts. The existing optimality criteria largely depend on estimated tensor/ODF/EAP and their invariant measures. In (iii), one needs to use some optimization algorithms to solve $\hat{\mathbf{q}}_{i} i_{n}=\operatorname{argmin} L\left(\begin{array}{cc}\mathbf{q}_{i} & i_{n}\end{array}\right)$. Since the optimality criterion may not be convex, calculating $\hat{\mathbf{q}}_{i} i_{n}{ }_{n}$ is not a trivial problem at all.

As an illustration, we consider the reconstruction of a diffusion tensor field, denoted by $\beta(\mathbf{v}): \mathbf{v} \quad \mathcal{V}$, based on model (4.37). We consider the covariance matrix of $\hat{\beta}(\mathbf{v})$ at voxel $\mathbf{v}$, denoted by $C(\hat{\beta}(\mathbf{v}))$. As shown in $(165), C(\hat{\beta}(\mathbf{v}))$ depends on the SNR, the $b$ value, the number of of baseline acquisitions, denoted by $m$, the diffusion tensor matrix, and the gradient encoding scheme. Let $p(\beta(\mathbf{v}))$ be the prior distribution of $\beta(\mathbf{v})$, which may represent prior knowledge of the underlying fiber orientations of the tissue being imaged. A Bayesian criterion function can be written as

$$
\operatorname{GSI}(m, b, \mathrm{SNR}, \mathbf{x})=\int \Psi C(\hat{\beta}(\mathbf{v})) p(\beta(\mathbf{v})) d \beta(\mathbf{v}),
$$

where $\Psi$ is a pre-specified function, such as the trace. We can use GSI as an index to compare different DT acquisition schemes.

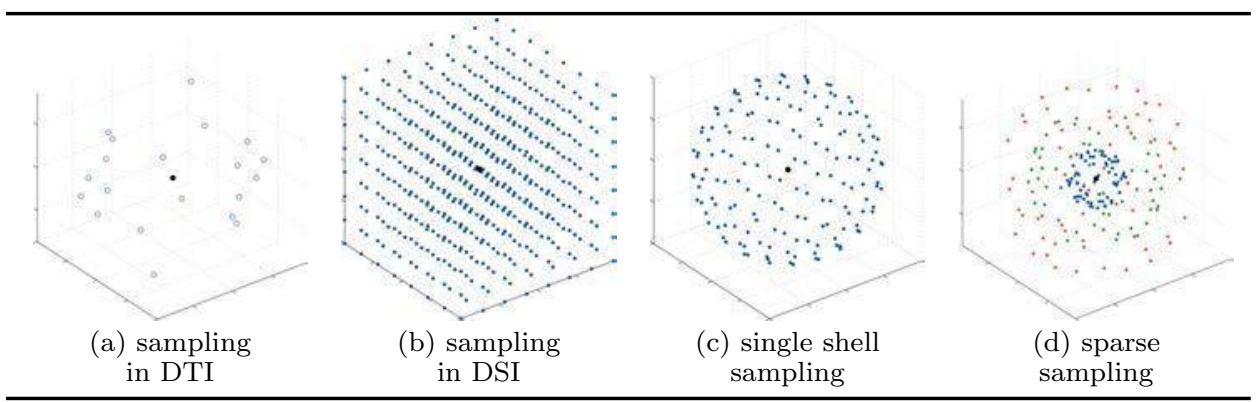

\section{FIGURE 4.10}

Several kinds of sampling in $\mathbf{q}$-space. The black dot in $\mathbf{q}=(0,0,0)^{T}$ is the baseline image without diffusion gradient. Note that although we showed sampling in $\mathbb{R}^{3}$, normally only samples in a half space are used, e.g., $(0,0,1) \mathbf{q}=q_{z} \quad 0$. (a) Sampling used in DTI, normally less than 20 DWI images are used; (b) dense Cartesian sampling used in DSI. Note in practice the Cartesian samples inside a given Ball are used. (c) Single shell sampling used in sHARDI methods, e.g., QBI, DOT etc. (d) Sparse sampling used in mHARDI methods, e.g., DPI, SHORE, SPFI. Note although normally multiple-shell sampling is used, any sampling scheme can be used in mHARDI methods. 
TABLE 4.1

The condition numbers and gradient sampling indices (GSI) of thirteen acquisition schemes in (123).

\begin{tabular}{lccccc}
\hline \hline $\begin{array}{l}\text { Scheme } \\
\text { name }\end{array}$ & $\begin{array}{c}\text { Condition } \\
\text { number }\end{array}$ & $\begin{array}{c}\text { Number of } \\
\text { directions }\end{array}$ & $m$ & Repetition & $\begin{array}{c}\text { GSI }(m, 900,15, \mathbf{x}) \\
\times 10^{7}\end{array}$ \\
\hline Tetrahedral & 9.148 & 6 & 1 & 20 & 2.944 \\
Cond6 & 5.989 & 6 & 1 & 20 & 1.398 \\
Decahedral & 2.749 & 10 & 2 & 12 & 0.8080 \\
Jones noniso & 2.562 & 7 & 1 & 18 & 0.8083 \\
Dual-gradient & 2.000 & 6 & 1 & 20 & 0.7052 \\
Jones10 & 1.624 & 10 & 2 & 12 & 0.5865 \\
Jones20 & 1.615 & 20 & 3 & 6 & 0.6177 \\
Jones30 & 1.595 & 30 & 5 & 4 & 0.5886 \\
Papadakis & 1.587 & 12 & 2 & 10 & 0.5269 \\
Jones6 & 1.583 & 6 & 1 & 20 & 0.5954 \\
Muthupallai & 1.581 & 6 & 1 & 20 & 0.5829 \\
Tetraortho & 1.528 & 7 & 1 & 18 & 0.5127 \\
DSM & 1.323 & 6 & 1 & 20 & 0.6014 \\
\hline \hline
\end{tabular}

To compare the adequacy of differing image acquisition strategies, we calculated the values of GSI for thirteen data acquisition schemes that were used previously to demonstrate the importance of the condition number in the determination of noise characteristics for particular acquisition schemes (see Tables 1 and 2 of (123)). Table 4.6 presents the GSI values for each of the thirteen acquisition schemes at $b=900 \mathrm{~s} / \mathrm{mm}^{2}$. Although the DSM scheme in (123) has the smallest condition number, its GSI was larger than those of the Papadakis and Tetraortho schemes. In terms of both the number of images and GSI, it seems that the Papadakis scheme is the best among these thirteen strategies.

There are many open questions in the design of sampling mechanisms for various HARDI models.

Little has been done on the design of effective sampling mechanisms for various HARDI models. Several key difficulties include the choice of $L\left(\begin{array}{lll}\mathbf{q}_{i} & i & n\end{array}\right)$ and its optimization, particularly for a multiple q-shell acquisition.

Nothing has been done on the use of the uncertainty of estimated fiber tracks to design sampling mechanism. Since our primary objective of interest is to reconstruct fiber tracks, it is important to develop some design criterion based on the uncertainty of estimated fiber tracks. As discussed above, since there is a lack of theoretical results on the uncertainty of estimated fiber tracks, it is impossible to develop an optimal sampling mechanism based on such results.

\subsection{Registration}

The above sections focus on estimation of the diffusion signal, EAP and ODF for an individual subject. Spatial alignment, also called image registration, is an important issue for group analysis. Image registration has been studied for decades in the medical image analysis domain, and in the last ten years some methods have been proposed to align DWI 
data, or the estimated tensor/ODF/EAP data. Although image registration techniques in dMRI originate from vector-valued image registration methods, these are not applicable to directly apply vector-valued image registration methods to diffusion data (4). An image registration method for diffusion data normally includes two aspects. One is the spatial alignment of 3D anatomical structures, and the other one is the re-orientation of local diffusion profiles. Some methods propose to perform these two steps separately for diffusion data $(4,73,33,112,149)$, i.e. perform re-orientation after spatial alignment. Some other methods propose to consider the re-orientation issue inside the cost function of the registration and perform these two steps iteratively $(29,42,153,67,150,56,161)$.

Reference (4) first addressed the re-orientation issue in dMRI and proposed "finite strain" (FS) and "preservation of principal direction" (PPD) to orient the tensor image, where PPD gives the best performance. The idea of PPD is to keep the tensor shape while rotating the principal direction of the tensor using the Jacobian matrix of the deformation field, and the idea of FS is to rotate the tensor using a rotate matrix extracted from the Jacobian matrix of the deformation field. It was shown that FS cannot consider shearing or scaling effect of transforms (4). PPD is widely used in tensor registration $(29,42,33)$. Re-orientation of the DWI signal, ODF and EAP data is more complicated compared with tensor data. Since the FS method is much simpler than PPD, some HARDI methods use FS to orient the ODF or EAP represented by the SH basis due to the closed form of rotation of the SH basis $(26,67)$. Some other methods separate diffusion signals and ODFs using some kind of basis functions, then re-orientate the basis function separately and combine the re-orientated functions together $(73,112,161)$. The basis functions in (73) are delta functions, and those in $(52,112,161)$ are fiber response functions.

There are some open questions in the registration of diffusion data.

Does the registration need to be performed in diffusion signals or estimation results (tensors, ODFs, or EAPs)? Most methods perform registration on tensors, ODFs, or EAPs, because the estimation results are more spatially meaningful than DWI signals. However these methods are largely affected by the reconstruction methods used. Some registration methods were performed directly in DWI signals. However, these registration methods still need to consider a model for re-orientation DWI signals.

How can re-orientation be done? It is well accepted that registration of diffusion data needs re-orientation. However, there is no consistent and well-accepted way to do re-orientation. PPD is well accepted for tensor data, which assumes that the shape of the local diffusion profile does not change. However, the current state-of-the-art registration methods in HARDI changes the shape of local diffusion profiles for re-orientation (73, $52,112,161)$.

It is critical to develop a reliable evaluation and validation system for registration algorithms. Unfortunately, little has ever been done on such development due to many fundamental difficulties, such as the two open questions discussed above.

\subsection{Group Analysis}

In the current literature, there exist three major approaches to the group analysis of diffusion imaging data: region-of-interest (ROI) analysis, voxel-based analysis, and fiber tract- based analysis (124, 103, 125). 
The ROI analysis can be performed by registering individual subject DWI images to an atlas and then averaging diffusion properties in some manually drawn ROIs of the atlas (125). Subsequently, a group analysis can be carried out to correlate all statistics at each ROI or across multiple ROIs with covariates of interest. An advantage of ROI analysis is that processing is relatively simple and robust against imperfect registration. The three drawbacks of ROI analysis include the difficulty in identifying meaningful ROIs, particularly the long curved structures common in fiber tracts, the instability of statistical results obtained from ROI analysis, and the partial volume effect in relatively large ROIs $(70,164)$. A stringent assumption of ROI analysis is that diffusion properties in all voxels of the same ROI are essentially homogeneous, which is largely false for dMRI data. Moreover, this form of analysis leads to limited localization of findings.

Voxel-based analysis has been widely used in neuroimaging studies. It involves registering each subject into a study-specific reference space and fitting a statistical model to the smoothed and registered diffusion property imaging data from multiple subjects at each voxel to generate a parametric map of test statistics (or $p$-values). Subsequently, a multiple-comparison procedure such as false discovery rate is applied to correct for multiple comparisons across the many voxels of the imaging volume (146). The major drawbacks of voxel-based analysis include poor alignment quality and the arbitrary choice of smoothing extent $(124,80)$. Moreover, in practice, one has to interpret the findings based on voxelwise comparison of the eigenvector and/or tensor images with great caution $(120,162)$, since they are very sensitive to alignment inaccuracies compared with FA images.

Fiber tract-based analysis has received growing interest, since it may be more robust to alignment inaccuracies, while directly incorporating fiber tract information $(124,103,160$, $70,164,71)$. There are three major fiber tract-based analysis methods including tract-based spatial statistics (TBSS), medial sheet based analysis, and fiber tract analysis. A tract based spatial statistics (TBSS) framework was developed to construct local diffusion properties along the white matter skeleton and then perform pointwise hypothesis tests on the skeleton (124). However, TBSS does not have an explicit tract representation that can be uniquely linked to individual fibers throughout the brain, while the use of maximal FA values renders TBSS sensitive to DWI artifacts.

A medial model-based framework was developed for the statistical analysis of diffusion properties on the medial manifolds of fiber tracts followed by testing pointwise hypotheses on the medial manifolds (160). The framework consists of effectively modeling six sheetlike fasciculi by using deformable medial representations, averaging and combining tensorbased features along directions locally perpendicular to the tracts, and pointwise statistical analysis. However, it is limited to the sheet-like white matter tracts and relies on expertdriven segmentation of the fasciculi.

A fiber tract analysis framework was developed for the statistical analysis of diffusion properties along major fiber tracts followed by using functional data analysis $(70,164,138$, $66,69,104,53)$. See $(138)$ for an overview of the fiber tract analysis framework developed at UNC-Chapel Hill. The fiber tract analysis framework consists of using anatomically informed curvilinear regions to analyze diffusion at specific locations all along fiber tracts, taking weighted averages at each step along the fiber bundles, an unbiased atlas-building step, and a Functional Analysis of Diffusion Tensor Tract Statistics (FADTTS) pipeline. This form of analysis results in highly localized statistics that can be visualized back on the individual fiber bundles. Moreover, there is great interest in developing new fiber registration methods for group analysis $(65,57,169,140,79)$.

A set of FADTTS has been developed for delineating the structure of the variability of multiple diffusion properties or tensors along major white matter fiber bundles and their association with a set of covariates for both cross-sectional and longitudinal studies $(164,156,163,159,75,166)$. The advantages of FADTTS are that they are capable of 
modelling the structured inter-subject variability by a functional principal component analysis method, testing the joint effects by a global test statistic and local test statistics, and constructing simultaneous confidence bands of the interested effects through a resampling method. Statistically, as shown in various simulations and real data analysis, these statistical methods in FADTTS are much more powerful than the standard voxel-wise methods.

As an illustration, we applied FADTTS to study the spatial-temporal dynamics of whitematter fiber tracts in a clinical study of neurodevelopment. There are 298 high-quality scans available for 137 children with 83 males and 54 females. As a graphical illustration, FA measures were plotted along the genu and splenium of the corpus callosum for each subjects within each age group (Figure 4.11 (a)). FA measures were also plotted for 35 subjects along the genu tract (Figure 4.11 (b) and (c)). An obvious increasing trend for the values of FA were observed at nearly all grid points, especially from neonate to the first year.

For the genu tract, we fitted a functional mixed-effects model (FMEM) in (156) to the FA curves, denoted by $y_{i j}(s)$, from all 137 subjects. Specifically, FMEM is given by

$$
y_{i j}(s)=\mathbf{x}_{i j}^{T} B(s)+\mathbf{z}_{i j}^{T} \xi_{i}(s)+\eta_{i j}(s)+\epsilon_{i j}(s),
$$

where $\mathbf{x}_{i j}=\left(1, \operatorname{Dir}_{i j}, \mathrm{G}_{i}, \mathrm{Age}_{i j 1}, \mathrm{Age}_{i j 2}\right)^{T}, \mathbf{z}_{i j}=\left(1, \mathrm{Age}_{i j 1}, \mathrm{Age}_{i j 2}\right)^{T}$ and $\operatorname{Age}_{i j 1}\left(\mathrm{Age}_{i j 2}\right)$ is an indicator variable indicating whether a subject belongs to the first (second) year age group. The coefficient functions related to $\mathrm{Age}_{i j 1}$ and $\mathrm{Age}_{i j 2}$ can be used to investigate whether there is some change from neonate to the first year of life, from the first year to the second year, and from neonate to the second year. Moreover, in model $(4.49), \eta_{i j}(s)$ primarily characterizes within-curve spatial correlation structure, while $\xi_{i}(s)$ primarily characterizes the subject-level variations and within-subject spatial-temporal correlation. Then we estimated the functional coefficients. For hypothesis testing, we constructed the global test statistic to test the gender, number of gradient directions and age effects on FA values. We approximated the $p$ value of the global test using the resampling method with 5,000 replications. Finally, we constructed the $95 \%$ simultaneous confidence bands for the functional coefficients.

The hypothesis testing results show that there are significant age and number of gradient direction effects on FA, RD and AD values. The FA are significantly different between neonate versus the first year, and between the first year versus the second year with $p$ value $<.0001$, far smaller than a 0.05 significance level. It is observed from Figure 4.11 (b) that mean FA values increase from neonate to the first year and then from the first year to the second year. Moreover, the change from the neonate to the first year is larger than that from the first year to the second year. No gender difference in FA was found for the genu tract.

There are many open questions in the joint analysis of diffusion imaging data and other data.

All fiber tract-based methods including FADTTS are only applicable to these major white matter tracts in which one can establish common localization across subjects. However, the centroid of the localization of white matter lesion could vary across time and subjects. In some heterogeneous populations, it is possible that tract-specific changes occur in only a subset of subjects. In these scenarios, none of group analysis methods discussed above would be appropriate.

There is an urgent demand for the development of functional regression methods for the analysis of repeated functional data and clinical data obtained from longitudinal and familial studies. Although there is a handful of papers on the development of statistical models and their estimation methods for repeated functional data, the methodology for dealing with such data is still in its infancy, and further computational and theoretical development is greatly needed. 

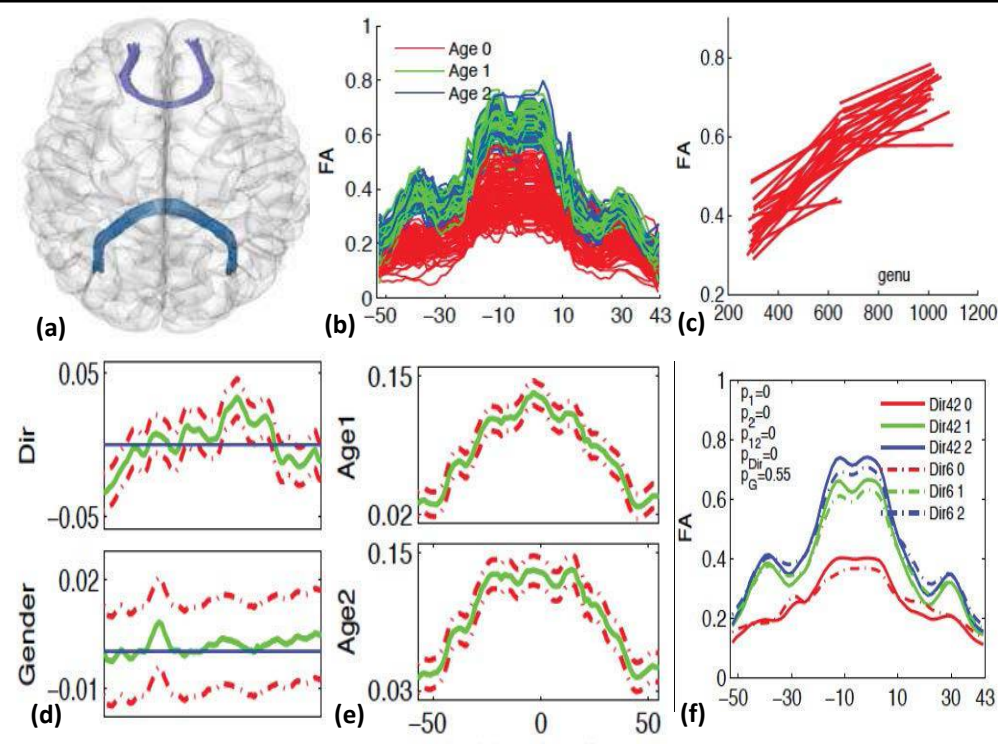

\section{FIGURE 4.11}

(a) The commissural bundles of the genu and splenium of the corpus callosum. (b) FA values along the genu tract for all 137 subjects in each age group. (c) FA values varying over age at a selected location along the genu tract. (d) and (e) 95\% simultaneous confidence bands for varying coefficient functions for FA along the genu of the corpus callosum tract. The solid curves are the estimated coefficient functions, and the dashed curves are the $95 \%$ confidence bands. The thin horizontal line is the line crossing the origin. (f) $p_{1}$ is the $p$ value for the difference in the diffusion measure between neonate and the first year, $p_{2}$ is the $p$ value for the difference in the diffusion measure between neonate and the second year, $p_{12}$ is the $p$ value for the difference between the first year and the second year, $p_{G}$ is the $p$ value for the gender effect, $p_{D i r}$ is the $p$ value for the effect of the number of gradient directions.

There is an urgent demand for the development of high-dimensional risk prediction models by integrating and identifying important white matter tracts, functional images, and biological markers for risk prediction. These models can have a great impact in public health from disease prevention, to detection, to treatment selection. For instance, it is interesting to consider generalized functional linear models, in which a scalar outcome (e.g., diagnostic group) is used as the response and fiber bundle diffusion properties are used as varying covariate functions (or functional predictor) $(114,117)$.

\subsection{Public Resources}

\subsubsection{Datasets}

Public datasets are important for reproducible research. For synthetic data simulation, people normally use a mixture of tensor models or cylinder models to generate the ground 
truth of signals (105), then corrupt the ground truth signal using Rician noise. Here are some real public datasets for simulation and evaluation.

Q1, Q2, Q3 data from HCP: http://www.humanconnectome.org/data/, three shells, staggered 90 direction per shell $(31), b=1000,2000,3000 \mathrm{~s} / \mathrm{mm}^{2}, 1.25 \mathrm{~mm}$ isotropic voxels.

HARDI data for Stanford: http://purl.stanford.edu/yx282xq2090, single shell HARDI, 150 directions, $b=2000 \mathrm{~s} / \mathrm{mm}^{2}$.

Phantom data for fiber cup 2009 (58): http://lnao.lixium.fr/spip.php?rubrique79, three shells, the same 64 directions with twice the scans per shell, $b=650,1500,2000 \mathrm{~s} / \mathrm{mm}^{2}$.

HARDI reconstruction challenge: http://hardi.epfl.ch/static/events/2013ISBI, 3 predetemined sampling schemes including DTI $\left(b=1200 \mathrm{~s} / \mathrm{mm}^{2}, 32\right.$ directions $)$, HARDI ( $b=3,000 \mathrm{~s} / \mathrm{mm}^{2}, 64$ directions $)$, and Heavyweight $\left(b<12,000 \mathrm{~s} / \mathrm{mm}^{2}, 515\right.$ acquisitions).

DWI datasets from the Alzheimer's Disease Neuroimaging Initiative (ADNI): http://www. adni-info.org/, 264 subjects with a total of 799 DWI datasets, $b=$ $1,000 \mathrm{~s} / \mathrm{mm}^{2}$, and 41 directions.

\subsubsection{Software}

There is a branch of open source codes and software for diffusion MRI data processing. Here are a recommendation list.

FSL: http://fsl.fmrib.ox.ac.uk/fsl/fslwiki/FDT/UserGuide. Eddy current correction, tensor estimation, multi-tensor estimation, registration of scalar images, deterministic tracking, probabilistic tracking, TBSS, QBI, SD.

3D Slicer: http://www.slicer.org/. DWI denoise, DTI, fiber tracking, visualization of tensors/fibers.

CAMINO: http://www.cs.ucl.ac.uk/research/medic/camino. DTI, multi-tensor estimation, QBI, SD, PASMRI, data simulation, tensor registration, uniform sampling scheme based on minimization of electrostatic energy, peak detection, visualization of tensors/ODFs/fibers/peaks.

MITK: http://www.mitk.org/. DTI, QBI, global fiber tracking, deterministic fiber tracking, peak detection, TBSS, data simulation, visualization of tensors/ODFs/fibers.

MRtrix: http://www.brain.org.au/software/mrtrix/. DTI, SD, deterministic/probabilistic fiber tracking, visualization of tensors/ODFs/fibers/peaks.

MRI Studio: https://www.mristudio.org. DTI, deterministic fiber tracking, visualization of tensors/fibers.

Trackvis: http://www.trackvis.org, DTI, QBI, DSI, fiber tracking, visualization of fibers.

MEDINRIA: http://med.inria.fr/. DTI, deterministic fiber tracking, visualization of tensors/fibers.

DTI-TK: http://dti-tk.sourceforge.net/pmwiki/pmwiki.php. Tensor estimation, tensor registration, image format conversion, visualization of tensors/fibers. 
Fibernavigator: http://scilus.github.io/fibernavigator/. Visualization of tensors/ODFs/ fibers/peaks.

DIPY: https://github.com/nipy/dipy. DTI, SD, QBI, DSI, fiber tracking, visualization of tensors/ODFs/fibers/peaks.

DSI-studio : http://dsi-studio.labsolver.org/. DTI, QBI, DSI, fiber tracking, visualization of ODFs/fibers.

SSPM: http://www.nitrc.org/projects/sspm/. Group analysis toolbox for carrying out voxel-based analysis and fiber tract-based analysis.

NAMICDTIFIBER: https://www.nitrc.org/projects/namicdtifiber/. UNC/Utah NAMIC DTI Fiber Analysis Framework for carrying out fiber tract analysis.

\subsection{Glossary}

ADC: Apparent Diffusion Coefficient

DOT: Diffusion Orientation Transform

DPI: Diffusion Propagator Imaging

DSI: Diffusion Spectrum Imaging

DTI: Diffusion Tensor Imaging

DWI: Diffusion Weighted Imaging

EAP: Ensemble Average Propagator

FA: Fractional Anisotropy

GDTI: Generalized Diffusion Tensor Imaging

GFA: Generalized Fractional Anisotropy

HARDI: High Angular Resolution Diffusion Imaging

HOT: High-Order Tensor

MD: Mean Diffusivity

ODF: Orientation Distribution Function

PGSE: Pulsed Gradient Spin-Echo

QBI: Q-Ball Imaging

SH: Spherical Harmonic

SHORE: Simple Harmonic Oscillator Reconstruction and Estimation

SNR: Signal-To-Noise ratio

SPFI: Spherical Polar Fourier Imaging 


\section{Bibliography}

[1] I. Aganj, C. Lenglet, G. Sapiro, E. Yacoub, K. Ugurbil, and N. Harel. Reconstruction of the orientation distribution function in single and multiple shell q-ball imaging within constant solid angle. Magnetic Resonance in Medicine, 2:554-566, 2010.

[2] S. Aja-Fernández, A. Tristán-Vega, and W.S. Hoge. Statistical noise analysis in GRAPPA using a parametrized noncentral Chi approximation model. Maqn Reson Med. 65:1195-1206, 2011.

[3] Santiago Aja-Fernández, Carlos Alberola-López, and C-F Westin. Noise and signal estimation in magnitude MRI and Rician distributed images: A LMMSE approach. Image Processing, IEEE Transactions on, 17(8):1383-1398, 2008.

[4] D. C. Alexander, C. Pierpaoli, P. J. Basser, and J. C. Gee. Spatial transformations of diffusion tensor magnetic resonance images. IEEE Transactions on Medical Imaaing. 20:1131-1139, 2001.

[5] Daniel C Alexander. Multiple-fiber reconstruction algorithms for diffusion MRI. $\underline{A n-}$ nals of the New York Academu of Sciences. 1064(1):113-133, 2005.

[6] A. W. Anderson. Theoretical analysis of the effects of noise on diffusion tensor imaging. Maanetic Resonance in Medicine. 46:1174-1188, 2001.

[7] A.W. Anderson. Measurement of fiber orientation distributions using high angular resolution diffusion imaging. Maqnetic Resonance in Medicine. 54(5):1194-1206, 2005.

[8] E. Arias-Castro, J. Salmon, and R. Willett. Oracle inequalities and minimax rates for nonlocal means and related adaptive kernel-based methods. SIAM J. Imaqing Sciences. 5:944-992, 2012.

[9] V. Arsigny, Fillard P, Pennec X, and Ayache N. Geometric means in a novel vector space structure on symmetric positive definite matrices. SIAM. J. Matrix Anal. Appl. 29:328-347, 2007.

[10] Y. Assaf, R.Z. Freidlin, G.K. Rohde, and P.J. Basser. New modeling and experimental framework to characterize hindered and restricted water diffusion in brain white matter. Maanetic Resonance in Medicine. 52(5):965-978, 2004.

[11] Haz-Edine Assemlal, David Tschumperlé, and Luc Brun. Efficient and robust computation of PDF features from diffusion MR signal. Medical Imaqe Analysis. 13:715-729, 2009 .

[12] A. C. Atkinson and A. N. Donev. Optimum Experimental Designs. Clarendon Press, Oxford, 1992.

[13] P. J. Basser, J. Mattiello, and D. LeBihan. Estimation of the effective self-diffusion tensor from the NMR spin echo. Journal of Maqnetic Resonance Series B. 103:247$254,1994$.

[14] P. J. Basser, J. Mattiello, and D. LeBihan. MR diffusion tensor spectroscopy and imaging. Biophusical Journal. 66:259-267, 1994. 
[15] Peter J. Basser and Derek K. Jones. Diffusion-tensor MRI: Theory, experimental design and data analysis - A technical review. NMR in Biomedicine. 15(7-8):456467, 2002.

[16] Peter J. Basser and Sinisa Pajevic. A normal distribution for tensor-valued random variables applications to diffusion tensor MRI. IEEE Transactions on Medical Imaqing. 22:785-794, 2003.

[17] Peter J. Basser and Carlo Pierpaoli. Microstructural and physiological features of tissues elucidated by quantitative-diffusion-tensor MRI. Journal of Maqnetic Resonance. 111:209-219, 1996.

[18] S. Becker, K. Tabelow, S. Mohammadi, N. Weiskopf, and J. Polzehl. Adaptive smoothing of multi-shell diffusion weighted magnetic resonance data by msPOAS. Technical report, Weierstrass-Institute, 2012.

[19] S. Becker, K. Tabelow, H.U. Voss, A. Anwander, R.M. Heidemann, and J. Polzehl. Position-orientation adaptive smoothing of diffusion weighted magnetic resonance data (POAS). Med. Imaqe Anal.. 16:1142-1155, 2012.

[20] SMA Becker, K. Tabelow, S. Mohammadi, N. Weiskopf, and J. Polzehl. Adaptive smoothing of multi-shell diffusion weighted magnetic resonance data by msPOAS. NeuroImage. 95:90-105, 2014.

[21] SMA Becker, Karsten Tabelow, Henning U. Voss, Alfred Anwander, Robin M. Heidemann, and Jörg Polzehl. Position-orientation adaptive smoothing of diffusion weighted magnetic resonance data (POAS). Medical Imaqe Analusis. 16(6):1142-1155, 2012.

[22] T.E. Behrens, M.W. Woolrich, M. Jenkinson, H. Johansen-Berg, R.G. Nunes, S. Clare, P.M. Matthews, J.M. Brady, and S.M. Smith. Characterization and propagation of uncertainty in diffusion-weighted mr imaging. Maan Reson Med.. 50:1077-1088, 2003.

[23] L. Beltrachini, N. von Ellenrieder, and C.H. Muravchik. Error bounds in diffusion tensor estimation using multiple-coil acquisition systems. Magn Reson Imaqing. 31:13721383, 2013.

[24] M. A. Bernstein, K. F. King, and X. Zhou, editors. Handbook of MRI Pulse Sequences. Elsevier Academic Press, 2004.

[25] D. Le Bihan, E. Breton, D. Lallemand, P. Grenier, E. Cabanis, and M. Laval-Jeantet. MR imaging of intravoxel incoherent motions: application to diffusion and perfusion in neurologic disorders. Radiologu. 161:401-407, 1986.

[26] Luke Bloy and Ragini Verma. Demons registration of high angular resolution diffusion images. In Biomedical Imaging: From Nano to Macro, 2010 IEEE International Symposium on, pages 1013-1016. IEEE, 2010.

[27] A. Buades, B. Coll, and J. M. Morel. Imaging denoising methods: A new nonlocal principle. SIAM Review. 52:113-147, 2010.

[28] P. Buhlmann and S. van de Geer. Statistics for High-Dimensional Data: Methods. Theory and Applications. Springer, 2011.

[29] Y. Cao, M.I. Miller, S. Mori, R.L. Winslow, and L. Younes. Diffeomorphic matching of diffusion tensor images. In Computer Vision and Pattern Recognition Workshop, 2006. CVPRW'06. Conference on, pages 67-67. IEEE, 2006. 
[30] O. Carmichael, J. Chen, D. Paul, and J. Peng. Diffusion tensor smoothing through weighted Karcher means. Electron. J. Stat.. 7:1913-1956, 2013.

[31] E. Caruyer, J. Cheng, C. Lenglet, G. Sapiro, T. Jiang, R. Deriche, et al. Optimal design of multiple Q-shells experiments for Diffusion MRI. In Computational Diffusion MRI - MICCAI Workshop, 2011.

[32] Emmanuel Caruyer. Q-space diffusion MRI: Acquisition and signal processing. PhD thesis, University College London, UK, 2012.

[33] Can Ceritoglu, Kenichi Oishi, Xin Li, Ming-Chung Chou, Laurent Younes, Marilyn Albert, Constantine Lyketsos, Peter van Zijl, Michael I Miller, and Susumu Mori. Multi-contrast large deformation diffeomorphic metric mapping for diffusion tensor imaging. Neuroimaqe. 47(2):618-627, 2009.

[34] L.C. Chang, D.K. Jones, and C. Pierpaoli. Restore: Robust estimation of tensors by outlier rejection. Maqn. Reson. Med.. 53:1088-1095, 2005.

[35] L.C. Chang, L. Walker, and C. Pierpaoli. Informed restore: A method for robust estimation of diffusion tensor from low redundancy datasets in the presence of physiological noise artifacts. Maqn. Reson. Med.. 68:1654-1663, 2012.

[36] C. Chefd'hotel, D. Tschumperlé, R. Deriche, and O. Faugeras. Regularizing flows for constrained matrix-valued images. Journal of Mathematical Imaqinq and Vision. 20(1-2):147-162, 2004.

[37] Jian Cheng, Rachid Deriche, Tianzi Jiang, Dinggang Shen, and Pew-Thian Yap. Nonnegative spherical deconvolution (NNSD) for estimation of fiber orientation distribution function in single-/multi-shell diffusion MRI. NeuroImage. 101:750-764, 2014.

[38] Jian Cheng, Rachid Deriche, Tianzi Jiang, Dinggang Shen, and Pew-Thian Yap. NonNegative Spherical Deconvolution (NNSD) for Fiber orientation distribution function estimation. In Computational Diffusion MRI and Brain Connectivitu. pages 81-93, 2014.

[39] Jian Cheng, Aurobrata Ghosh, Rachid Deriche, and Tianzi Jiang. Model-free, regularized, fast, and robust analytical orientation distribution function estimation. In Medical Imaqe Computing and Computer-Assisted Intervention - MICCAI. 13(Pt 1):648-656. 2010.

[40] Jian Cheng, Aurobrata Ghosh, Tianzi Jiang, and Rachid Deriche. Model-free and analytical EAP reconstruction via spherical polar fourier diffusion MRI. In Medical Image Computing and Computer-Assisted Intervention - MICCAI. 13(Pt 1):590597. 2010.

[41] Jian Cheng, Tianzi Jiang, and Rachid Deriche. Theoretical analysis and practical insights on EAP estimation via a unified HARDI framework. In Computational Diffusion MRI - MICCAI Workshop, 2011.

[42] Ming-Chang Chiang, Alex D. Leow, Andrea D. Klunder, Rebecca A. Dutton, Marina Barysheva, Stephen E. Rose, Katie L. McMahon, Greig I. De Zubicaray, Arthur W. Toga, and Paul M. Thompson. Fluid registration of diffusion tensor images using information theory. Medical Imaging, IEEE Transactions on, 27(4):442-456, 2008.

[43] R. D. Cook and S. Weisberg. Residuals and Influence in Regression. London: Chapman and Hall, 1982. 
[44] Marc-Alexandre Côté, Gabriel Girard, Arnaud Boré, Eleftherios Garyfallidis, JeanChristophe Houde, and Maxime Descoteaux. Tractometer: Towards validation of tractography pipelines. Medical Imaqe Analusis. 17(7):844-857, 2013.

[45] O. Coulon, D. C. Alexander, and S. Arridge. Diffusion tensor magnetic resonance image regularization. Medical Imaqe Analusis. 8:47-67, 2004.

[46] M. Descoteaux. High angular resolution diffusion MRI: From local estimation to segmentation and tractograpghy. PhD thesis, INRIA Sophia Antipolis, 2008.

[47] M. Descoteaux, E. Angelino, S. Fitzgibbons, and R. Deriche. Regularized, fast and robust analytical Q-ball imaging. Maqnetic Resonance in Medicine. 58:497-510, 2007.

[48] M. Descoteaux, R. Deriche, D.L. Bihan, J.F. Mangin, and C. Poupon. Multiple q-shell diffusion propagator imaging. Medical Image Analysis, 2010.

[49] M. Descoteaux, R. Deriche, T. R. Knösche, and A. Anwander. Deterministic and probabilistic tractography based on complex fiber orientation distributions. IEEE Transactions in Medical Imaaing. 28:269-286, 2008.

[50] M. Descoteaux, N. Wiest-Daesslé, S. Prima, C. Barillot, and R. Deriche. Impact of Rician adapted non-local means filtering on HARDI. Medical Image Computing and Computer-Assisted Intervention, 5242:122-130, 2008.

[51] M. Descoteaux, N. Wiest-Daessle, S. Prima, C. Barillot, and R. Deriche. Impact of Rician Adapted Non-local Means Filtering on HARDI. In MICCAI. 2008.

[52] T. Dhollander, W. Van Hecke, F. Maes, S. Sunaert, and P. Suetens. Spatial transformations of high angular resolution diffusion imaging data in q-space. In Computational Diffusion MRI - MICCAI Workshop, pages 73-83, 2010.

[53] C.Z. Di, C. M. Crainiceanu, B. S. Caffo, and Punjabi N. M. Multilevel functional principal component analysis. Annals of Applied Statistics. 3:458-488, 2009.

[54] I. L. Dryden, A. Koloydenko, and D. Zhou. Non-Euclidean statistics for covariance matrices, with applications to diffusion tensor imaging. Annals of Applied Statistics. 3:1102-1123., 2009.

[55] J. Du, A. Goh, S. Kushnarev, and A. Qiu. Geodesic regression on orientation distribution functions with its application to an aging study. NeuroImage. 87:416-426, 2014.

[56] Jia Du, Alvina Goh, and Anqi Qiu. Diffeomorphic metric mapping of high angular resolution diffusion imaging based on Riemannian structure of orientation distribution functions. Medical Imaging, IEEE Transactions on, 31(5):1021-1033, 2012.

[57] S. Durrleman, P. Fillard, X. Pennec, A. Trouvé, and N. Ayache. Registration, atlas estimation and variability analysis of white matter fiber bundles modeled as currents. NeuroImaqe. 55(3):1073-1090, 2011.

[58] P. Fillard, M. Descoteaux, A. Goh, S. Gouttard, B. Jeurissen, J. Malcolm, A. RamirezManzanares, M. Reisert, K. Sakaie, F. Tensaouti, et al. Quantitative evaluation of 10 tractography algorithms on a realistic diffusion MR phantom. NeuroImage. 2011. 
[59] P. Fillard, M. Descoteaux, A. Goh, S. Gouttard, B. Jeurissen, J. Malcolm, A. RamirezManzanares, M. Reisert, K. Sakaie, F. Tensaouti, T. Yo, J. F. Mangin, and C. Poupon. Quantitative evaluation of 10 tractography algorithms on a realistic diffusion MR phantom. NeuroImage. 56:220-234, 2011.

[60] P.T. Fletcher and S. Joshi. Riemannian geometry for the statistical analysis of diffusion tensor data. Signal Processing. 87:250-262, 2007.

[61] P.T. Fletcher, S. Venkatasubramanian, and S. Joshi. The geometric median on Riemannian manifolds with application to robust atlas estimation. NeuroImage. 45:S143S152, 2009.

[62] L. R. Frank. Characterization of anisotropy in high angular resolution dffusionweighted MRI. Maqnetic Resonance in Medicine. 47:1083-1099, 2002.

[63] O. Friman, G. Farneback, and C. F. Westin. A Bayesian approach for stochastic white matter tractography. IEEE Trans Med Imaging. 25:965-978, 2006.

[64] W. Gao, H.T. Zhu, and W. Lin. A unified optimization approach for diffusion tensor imaging technique. Neuroimaqe. 44(3):729-741, 2009.

[65] E. Garyfallidis, O. Ocegueda, D. Wassermann, and M. Descoteaux. Robust and efficient linear registration of white-matter fascicles in the space of streamlines. NeuroImage, 117, 2015.

[66] X. Geng, S. Gouttard, A. Sharma, H. Gu, M. Styner, and W. Lin. Quantitative tractbased white matter development from birth to age 2 years. NeuroImaqe. 61:542-557, 2012.

[67] X.J. Geng, T.J. Ross, H. Gu, W. Shin, W. Zhan, Y.P. Chao, C.P. Lin, N. Schuff, and Y. Yang. Diffeomorphic image registration of diffusion MRI using spherical harmonics. Medical Imaging, IEEE Transactions on, 30(3):747-758, 2011.

[68] I. Goh, C. Lenglet, P. M. Thompson, and R. Vidald. A nonparametric Riemannian framework for processing high angular resolution diffusion images and its applications to ODF-based morphometry. Neuroimaqe. 56:1181-1201, 2011.

[69] A.J. Goldsmith, C.M. Crainiceanu, B.S. Caffo, and D. Reich. Penalized functional regression analysis of white-matter tract profiles in multiple sclerosis. NeuroImage. $57: 431-439,2011$.

[70] C. B. Goodlett, P. T. Fletcher, J. H. Gilmore, and G. Gerig. Group analysis of DTI fiber tract statistics with application to neurodevelopment. NeuroImaqe. 45:S133S142, 2009.

[71] S. Greven, C. Crainiceanu, B. Caffo, and D. Reich. Longitudinal functional principal components analysis. Electronic Journal of Statistics. 4:1022-1054, 2010.

[72] P. Hagmann, L. Jonasson, P. Maeder, J.P. Thiran, Van J. Wedeen, and R. Meuli. Understanding Diffusion MR Imaging Techniques: From scalar diffusion-weighted imaging to diffusion tensor imaging and beyond. RadioGraphics. 26:S205-S223, 2006.

[73] Xin Hong, Lori R. Arlinghaus, and Adam W. Anderson. Spatial normalization of the fiber orientation distribution based on high angular resolution diffusion imaging data. Maanetic Resonance in Medicine. 61(6):1520-1527, 2009. 
[74] T. Hosey, G. Williams, and R. Ansorge. Inference of multiple fiber orientations in high angular resolution diffusion imaging. Maanetic Resonance in Medicine. 54(6):14801489, 2005.

[75] Z. W. Hua, D. B. Dunson, J. H. Gilmore, M. Styner, and H. T. Zhu. Semiparametric Bayesian local functional models for diffusion tensor tract statistics. NeuroImage. 63:460-674, 2012.

[76] P. J. Huber. Robust Statistics. Wiley Series in Probability and Statistics, 1981.

[77] S. Jbabdi and T. E Behrens. Long-range connectomics. Annals of the New York Academu of Sciences. 1305(1):83-93, 2013.

[78] S. Jbabdi and H. Johansen-Berg. Tractography: Where do we go from here? Brain Connect. 1:169-183, 2011.

[79] Y. Jin, Y. Shi, L. Zhan, B. A. Gutman, G. I. de Zubicaray, K. L. McMahon, M. J. Wright, A. W. Toga, and P. M. Thompson. Automatic clustering of white matter fibers in brain diffusion MRI with an application to genetics. NeuroImaqe. 100:75-90, 2014.

[80] D. K. Jones, M. R. Symms, M. Cercignani, and R. J. Howard. The effect of filter size on VBM analyses of DT-MRI data. NeuroImaqe. 26:546-554, 2005.

[81] D.K. Jones. Diffusion MRI: Theory, Methods, and Applications. Oxford University Press, Oxford, New York, 2011.

[82] D.K. Jones and M. Cercignani. Twenty-five pitfalls in the analysis of diffusion MRI data. NMR Biomed. 23:803-820, 2010.

[83] D.K. Jones, T.R. Knösche, and R. Turner. White matter integrity, fiber count, and other fallacies: The do's and don'ts of diffusion MRI. Neuroimaqe. 73:239-254, 2013.

[84] V. Katkovnik, A. Foi, K. Egiazarian, and J. Astola. From local kernel to nonlocal multiple-model image denoising. International Journal of Computer Vision. 86:1-32, 2010.

[85] Y. Kim, P.M. Thompson, A.W. Toga, L. Vese, and L. Zhan. HARDI denoising: Variational regularization of the spherical apparent diffusion coefficient SADC. Information Processing in Medical Imaaing. pages 515-527, 2009.

[86] V. Koltchinskii, L. Sakhanenko, and S. Cai. Integral curves of noisy vector fields and statistical problems in diffusion tensor imaging: Nonparametric kernel estimation and hypotheses testing. The Annals of Statistics. pages 1576-1607, 2007.

[87] B.W. Kreher, J.F. Schneider, I. Mader, E. Martin, J. Hennig, and K.A. Il'yasov. Multitensor approach for analysis and tracking of complex fiber configurations. Maanetic Resonance in Medicine. 54(5):1216-1225, 2005.

[88] P.W. Kuchel, A. Coy, and P. Stilbs. NMR "diffusion-diffraction" of water revealing alignment of erythrocytes in a magnetic field and their dimensions and membrane transport characteristics. Magnetic Resonance in Medicine. 37(5):637-643, 1997.

[89] Mariana Lazar. Mapping brain anatomical connectivity using white matter tractography. NMR in Biomedicine. 23(7):821-835, 2010. 
[90] Y. Li, H. Zhu, D. Shen, W. Lin, J. H. Gilmore, and J. G. Ibrahim. Multiscale adaptive regression models for neuroimaging data. Journal of the Roual Statistical Societu: Series B. 73:559-578, 2011.

[91] C. Liu, R. Bammer, B. Acar, and M. E. Moseley. Characterizing non-Gaussian diffusion by using generalized diffusion tensors. Magnetic Resonance in Medicine. 51:925937, 2004.

[92] C. Liu, R. Bammer, and M. E. Moseley. Generalized diffusion tensor imaging (GDTI): A method for characterizing and imaging diffusion anisotropy caused by non-Gaussian diffusion. Israel Journal of Chemistru. 43:145-154, 2003.

[93] M. Liu, B.C. Vemuri, and R. Deriche. A robust variational approach for simultaneous smoothing and estimation of DTI. NeuroImaqe. 67:33-41, 2013.

[94] J-F. Mangin, P. Fillard, Y. Cointepas, D. Le Bihan, V. Frouin, and C. Poupon. Toward global tractography. NeuroImage. 80:290-296, 2013.

[95] J. Marcinkiewicz. Sur une propriété de la loi de gauss. Mathematische Zeitschrift. 44(1):612-618, 1939.

[96] II. Maximov, F. Grinberg, and N.J. Shah. Robust tensor estimation in diffusion tensor imaging. J. Maan. Reson.. 213:136-144, 2011.

[97] T. McGraw, B.C. Vemuri, Y Chen, M. Rao, and T. Mareci. DT-MRI denoising and neuronal fiber tracking. Medical Image Analusis. 8(2):95-111, 2004.

[98] S. Merlet. Compressive sensing in diffusion MRI. PhD thesis, University of NiceSophia Antipolis, 2013.

[99] C.C. Moraga, C. Lenglet, R. Deriche, and J. Ruiz-Alzola. A Riemannian approach to anisotropic filtering of tensor fields. Signal Process, 87:217-352, 2007.

[100] S. Mori and P. C. M. van Zijl. Fiber tracking: principles and strategies: A technical review. NMR in Biomedicine. 15:468-480, 2002.

[101] S. Mori, S. Wakana, P.C.M. van Zijl, and L.M. Nagae-Poetscher. MRI Atlas of Human White Matter. Am Soc Neuroradiology, 2005.

[102] M.E. Moseley, Y. Cohen, J. Mintorovitch, J. Kucharczyk, J. Tsuruda, P. Weinstein, and D. Norman. Evidence of anisotropic self-diffusion. Radiologu. 176:439-445, 1990.

[103] L.J. O'Donnell, C.-F. Westin, and A.J. Golby. Tract-based morphometry for white matter group analysis. Neuroimaqe. 45:832-844, 2009.

[104] E. Özarslan, CG Koay, T. Shepherd, S. Blackband, and PJ Basser. Simple harmonic oscillator based reconstruction and estimation for three-dimensional q-space MRI. In International Society for Magnetic Resonance in Medicine (ISMRM), page 1396, 2009 .

[105] E. Özarslan, T. M. Shepherd, B. C. Vemuri, S. J. Blackband, and T. H. Mareci. Resolution of complex tissue microarchitecture using the diffusion orientation transform (DOT). NeuroImaqe. 31:1086-1103, 2006.

[106] E. Özarslan, B.C. Vemuri, and T.H. Mareci. Generalized scalar measures for diffusion MRI using trace, variance, and entropy. Maanetic Resonance in Medicine. 53(4):866$876,2005$. 
[107] N. G. Papadakis, D. Xing, C.L.H. Huang, L.D. Hall, and T. A. Carpenter. A comparative study of acquisition schemes for diffusion tensor imaging using MRI. Journal of Magnetic Resonance. 137(1):67-82, 1999.

[108] X. Pennec, P. Fillard, and N. Ayache. A Riemannian framework for tensor computing. International Journal of Computer Vision. 66:41-66, 2006.

[109] C. Pierpaoli and P. Basser. Toward a quantitative assessment of diffusion anisotropy. Magnetic Resonance in Medicine. 36:893-906, 1996.

[110] J. Polzehl and V. G. Spokoiny. Adaptive weights smoothing with applications to image restoration. J.R. Statist. Soc. B. 62:335-354, 2000.

[111] C. Poupon. Détection des faisceaux de fibres de la substance blanche pour l'étude de la connectivité anatomique cérébrale. PhD thesis, Ecole Nationale Supérieure des Télécommunications, December 1999.

[112] David Raffelt, J. Tournier, Jurgen Fripp, Stuart Crozier, Alan Connelly, Olivier Salvado, et al. Symmetric diffeomorphic registration of fibre orientation distributions. NeuroImage. 56(3):1171-1180, 2011.

[113] A. Raj, C. Hess, and P. Mukherjee. Spatial HARDI: Improved visualization of complex white matter architecture with Bayesian spatial regularization. Neuroimaqe. 54:396409, 2011.

[114] J. O. Ramsay and B. W. Silverman. Functional Data Analysis. Springer-Verlag, New York, 2005.

[115] S. Rao, J. G. Ibrahim, G. Cheng, P.T. Yap, and H.T. Zhu. SR-HARDI: Spatially regularizing high angular resolution diffusion imaging. Journal of Computational and Graphical Statistics, page in press, 2016.

[116] M. Reisert, I. Mader, C. Anastasopoulos, M. Weigel, S. Schnell, and V. Kiselev. Global fiber reconstruction becomes practical. NeuroImage. 54:955-962, 2011.

[117] P.T. Reiss and R.T. Ogden. Functional generalized linear models with images as predictors. Biometrics. 66:61-69, 2010.

[118] L. Sakhanenko. Numerical issues in estimation of integral curves from noisy diffusion tensor data. Statistics \&s Probabilitu Letters. 82(6):1136-1144, 2012.

[119] A. Schwartzman. Random ellipsoids and false discovery rates: Statistics for diffusion tensor imaging data. Ph.D. thesis, Stanford University, 2006.

[120] A. Schwartzman, W. Mascarenhas, and J. E. Taylor. Inference for eigenvalues and eigenvectors of Gaussian symmetric matrices. Ann. Statist.. 36:1423-1431, 2008.

[121] X. Shi, M. Styner, J. Lieberman, J. G. Ibrahim, W. Lin, and H. T. Zhu. Intrinsic regression models for manifold-valued data. Medical Image Computing and ComputerAssisted Intervention-MICCAI. pages 192-199, 2009.

[122] J. Sijbers, A.J. den Dekker, P. Scheunders, and D. Van Dyck. Maximum-likelihood estimation of Rician distribution parameters. IEEE Transactions on Image Processing. 17:357-361, 1998. 
[123] S. Skare, M. Hedehus, M. E Moseley, and T. Q. Li. Condition number as a measure of noise performance of diffusion tensor data acquisition schemes with MRI. Journal of Manetic Resonance. 147(2):340-352, 2000.

[124] S. M. Smith, M. Jenkinson, H. Johansen-Berg, D. Rueckert, T. E. Nichols, C. E. Mackay, K. E. Watkins, O. Ciccarelli, M.Z. Cader, P.M. Matthews, and T. E. Behrens. Tract-based spatial statistics: Voxelwise analysis of multi-subject diffusion data. $\mathrm{Neu}$ roImaqe. 31:1487-1505, 2006.

[125] L. Snook, C. Plewes, and C. Beaulieu. Voxel based versus region of interest analysis in diffusion tensor imaging of neurodevelopment. NeuroImage. 34:243-252, 2007.

[126] E.O. Stejskal and J.E. Tanner. Spin diffusion measurements: Spin echoes in the presence of a time-dependent field gradient. The Journal of Chemical Phusics. 42:288$292,1965$.

[127] Karsten Tabelow, Jörg Polzehl, Vladimir Spokoiny, and Henning U. Voss. Diffusion tensor imaging: Structural adaptive smoothing. NeuroImaqe. 39(4):1763-1773, 2008.

[128] Robert Tibshirani. Regression shrinkage and selection via the lasso. J. Roy. Statist. Soc. Ser. B, 58(1):267-288, 1996.

[129] J. Tournier, F. Calamante, and A. Connelly. Robust determination of the fibre orientation distribution in diffusion MRI: Non-negativity constrained super-resolved spherical deconvolution. NeuroImaqe. 35(4):1459-1472, 2007.

[130] J.D. Tournier, F. Calamante, D. Gadian, and A. Connelly. Direct estimation of the fiber orientation density function from diffusion-weighted MRI data using spherical deconvolution. NeuroImaqe. 23:1176-1185, 2004.

[131] A. Tristán-Vega, C.F. Westin, and S. Aja-Fernández. Estimation of fiber orientation probability density functions in high angular resolution diffusion imaging. NeuroImage. 47(2):638-650, 2009.

[132] Antonio Tristán-Vega and Santiago Aja-Fernández. DWI filtering using joint information for DTI and HARDI. Medical Imaqe Analusis. 14:205-218, 2010.

[133] Antonio Tristán-Vega, Carl-Fredrik Westin, and Santiago Aja-Fernández. A new methodology for the estimation of fiber populations in the white matter of the brain with the Funk-Radon transform. NeuroImaqe. 49:1301-1315, 2010.

[134] D. S. Tuch. Q-ball imaging. Maqnetic Resonance in Medicine. 52:1358-1372, 2004.

[135] D.S. Tuch. Diffusion MRI of Complex Tissue Structure. PhD thesis, MIT, 2002.

[136] D.S. Tuch, T.G. Reese, M. R. Wiegell, N. Makris, J.W. Belliveau, and Van J. Wedeen. High angular resolution diffusion imaging reveals intravoxel white matter fiber heterogeneity. Maanetic Resonance in Medicine. 48:577-582, 2002.

[137] D.S. Tuch, R.M. Weisskoff, J.W. Belliveau, and V.J. Wedeen. High angular resolution diffusion imaging of the human brain. In Proceedings of the 7th Annual Meeting of ISMRM, 1999.

[138] Audrey R Verde, Francois Budin, Jean-Baptiste Berger, Aditya Gupta, Mahshid Farzinfar, Adrien Kaiser, Mihye Ahn, Hans Johnson, Joy Matsui, Heather C Hazlett, et al. UNC-Utah NA-MIC framework for DTI fiber tract analysis. Frontiers in Neuroinformatics, 7, 2013. 
[139] E.A.H. von dem Hagen and R. M. Henkelman. Orientational diffusion reflects fiber structure within a voxel. Magnetic Resonance in Medicine. 48:454-459, 2002.

[140] D. Wassermann, Y. Rathi, S. Bouix, M. Kubicki, R. Kikinis, M. Shenton, and C. Westin. White matter bundle registration and population analysis based on Gaussian processes. In Information Processing in Medical Imaqina pages 320-332. Springer, 2011.

[141] V. J. Wedeen, D.L. Rosene, R. Wang, G. Dai, F. Mortazavi, P. Hagmann, J. H. Kaas, and W.Y.I. Tseng. The geometric structure of the brain fiber pathways. Science. 335:1628-1634, 2012.

[142] Van J. Wedeen, P. Hagmann, W.Y. I. Tseng, T. G. Reese, and R. M. Weisskoff. Mapping complex tissue architecture with diffusion spectrum magnetic resonance imaging. Maqnetic Resonance In Medicine. 54:1377-1386, 2005.

[143] C. F. Westin, S.E. Maier, H. Mamata, A. Nabavi, F.A. Jolesz, and R. Kikinis. Processing and visualization for diffusion tensor MRI. Medical Imaqe Analusis. 6:93-108, 2002.

[144] B. Whitcher, J. J. Wisco, N. Hadjikhani, and D. S. Tuch. Statistical group comparison of diffusion tensors via multivariate hypothesis testing. Magnetic Resonance in Medicine. 57:1065-1074, 2007.

[145] N. Wiest-Daesslé, S. Prima, P. Coupé, S. P. Morrissey, and C. Barillot. Non-local means variants for denoising of diffusion-weighted and diffusion tensor MRI. In Medical Imaqe Computing and Computer-Assisted Intervention-MICCAI 200\% pages 344351. Springer, 2007.

[146] K. J. Worsley, J. E. Taylor, F. Tomaiuolo, and J. Lerch. Unified univariate and multivariate random field theory. NeuroImaqe. 23:189-195, 2004.

[147] Y.C. Wu and A. L. Alexander. Hybrid diffusion imaging. NeuroImage. 36:617-629, 2007.

[148] Yu-Chien Wu, Aaron S. Field, and Andrew L. Alexander. Computation of diffusion function measures in q-space using magnetic resonance hybrid diffusion imaging. $\underline{I E E E}$ Transactions On Medical Imaqing. 27:858-865, 2008.

[149] P. Yap, G. Wu, H.T. Zhu, W. Lin, and D. Shen. Timer: Tensor image morphing for elastic registration. NeuroImage. 47:549-563, 2009.

[150] Pew-Thian Yap, Yasheng Chen, Hongyu An, Yang Yang, John H Gilmore, Weili Lin, and Dinggang Shen. Sphere: Spherical harmonic elastic registration of HARDI data. NeuroImaqe. 55(2):545-556, 2011.

[151] L. P. Yaroslavsky. Diaital Picture Processing. volume 9 of Springer Ser. Inform. Sci. Springer, Berlin, 1985.

[152] A. Yendikia, K. Koldewynb, S. Kakunooria, N. Kanwisherb, and B. Fischla. Spurious group differences due to head motion in a diffusion MRI study. NeuroImaqe. 88:79-90, 2014.

[153] B.T.T. Yeo, T. Vercauteren, P. Fillard, J.M. Peyrat, X. Pennec, P. Golland, N. Ayache, and O. Clatz. DT-REFinD: Diffusion tensor registration with exact finite-strain differential. Medical Imaging, IEEE Transactions on, 28(12):1914-1928, 2009. 
[154] T. Yu and P. Li. Spatial shrinkage estimation of diffusion tensors on diffusion weighted imaging data. Journal of the American Statistical Association. 108:864-875, 2013.

[155] T. Yu, C. Zhang, A. L. Alexander, and R. J. Davidson. Local tests for identifying anisotropic diffusion areas in human brain with DTI. Annals of Applied Statistics. 7:201-225, 2013.

[156] Y. Yuan, J. H. Gilmore, X. Geng, M. Styner, K. Chen, J. L. Wang, and H. T. Zhu. FMEM: Functional mixed effects modeling for the analysis of longitudinal white matter tract data. NeuroImage. 84:753-764, 2014.

[157] Y. Yuan, H. Zhu, W. Lin, and J. S. Marron. Local polynomial regression for symmetric positive definite matrices. Journal of Royal Statistical Society B. 74:697-719, 2012.

[158] Y. Yuan, H. T. Zhu, J. Ibrahim, W.L. Lin, and B.G. Peterson. A note on bootstrapping uncertainty of diffusion tensor parameters. IEEE Transactions on Medical Imaqing. 27:1506-1514, 2008.

[159] Y. Yuan, H.T. Zhu, M. Styner, J. H. Gilmore, and J. S. Marron. Varying coefficient model for modeling diffusion tensors along white matter bundles. Annals of Applied Statistics. 7:102-125, 2013.

[160] P. A. Yushkevich, H. Zhang, T.J. Simon, and J. C. Gee. Structure-specific statistical mapping of white matter tracts. Neuroimaqe. 41:448-461, 2008.

[161] Pei Zhang, Marc Niethammer, Dinggang Shen, and Pew-Thian Yap. Large deformation diffeomorphic registration of diffusion-weighted images with explicit orientation optimization. In Medical Image Computing and Computer-Assisted InterventionMICCAI 2013, pages 27-34. Springer, 2013.

[162] H. T. Zhu, Y. S. Cheng, J. G. Ibrahim, Y. M. Li, C. Hall, and W. L. Lin. Intrinsic regression models for positive definitive matrices with applications in diffusion tensor images. Journal of the American Statistical Association. 104:1203-1212, 2009.

[163] H. T. Zhu, R. Z. Li, and L. L. Kong. Multivariate varying coefficient model for functional responses. Annals of Statistics. 40:2634-2666, 2012.

[164] H. T. Zhu, M. Styner, N. S. Tang, Z. X. Liu, W. L. Lin, and J.H. Gilmore. FRATS: Functional regression analysis of DTI tract statistics. IEEE Transactions on Medical Imaaing. 29:1039-1049, 2010.

[165] H. T. Zhu, H. P. Zhang, J. G. Ibrahim, and B. G. Peterson. Statistical analysis of diffusion tensors in diffusion-weighted magnetic resonance image data (with discussion). Journal of the American Statistical Association. 102:1085-1102, 2007.

[166] H.T. Zhu, L. Kong, R. Li, M. Styner, G. Gerig, W. Lin, and J. H. Gilmore. Fadtts: functional analysis of diffusion tensor tract statistics. NeuroImaqe. 56:1412-1425, 2011.

[167] H.T. Zhu, Y.M. Li, J. G. Ibrahim, X.Y. Shi, and B.S. Peterson. Rician regression models for magnetic resonance images. Journal of the American Statistical Association. 104:623-637, 2009.

[168] V. Zipunnikov, B.S. Caffo, D.M. Yousem, C. Davatzikos, B.S. Schwartz, and C. Crainiceanu. Functional principal components model for high-dimensional brain imaging. NeuroImage. 58:772-784, 2011. 
[169] O. Zvitia, A. Mayer, R. Shadmi, S. Miron, and H. K. Greenspan. Co-registration of white matter tractographies by adaptive-mean-shift and Gaussian mixture modeling. IEEE Transactions on Medical Imaaing. 29(1):132-145, 2010. 
Taylor \& Francis Taylor \& Francis Group http://taylorandfrancis.com 